\title{
APPENDIX
}

\section{Characterization of Intersubunit Communication in the Virginiamycin trans-Acyl Transferase Polyketide Synthase}

Jonathan Dorival, ${ }^{\dagger}$ Thibault Annaval, ${ }^{\dagger}$ Fanny Risser, ${ }^{\dagger}$ Sabrina Collin, ${ }^{\dagger}$ Pierre Roblin, Christophe Jacob, ${ }^{\dagger}$ Arnaud Gruez, ${ }^{*}{ }^{\dagger}$ Benjamin Chagot ${ }^{*,+}$ and Kira J. Weissman ${ }^{*}{ }^{+}$

†UMR 7365, Ingénierie Moléculaire et Physiopathologie Articulaire (IMoPA), CNRS-Université de Lorraine, Biopôle de l'Université de Lorraine, Campus Biologie Santé, 9 Avenue de la Forêt de Haye, CS 50184, 54505 Vandœuvre-lès-Nancy Cedex, France

${ }^{\ddagger}$ Synchrotron SOLEIL, L'Orme des Merisiers, Saint-Aubin BP 48, 91192 Gif-sur-Yvette CEDEX, France §UR1268 Biopolymères, Interactions Assemblages (BIA), INRA, Rue de la Géraudière BP 71627, 44316 NANTES CEDEX 3, France 
We aimed to strengthen the case that our observations concerning the VirA ${ }^{C} D D /$ VirFG ${ }^{N} D D$ interface are generalizable to other trans-AT PKSs. For this, we benchmarked several web-based servers for the in silico identification of IDPs and protein-protein interaction regions (IUPred/ANCHOR ${ }^{1,2}$, PONDR $^{3,4}$, DisoPred ${ }^{5}$ and DisEMBL ${ }^{6}$ ) against VirA ${ }^{C} D D$ and VirFG ${ }^{N} D D$, to determine if they accurately predicted their respective behaviors. These approaches have complementary principles of operation (they are based either on alignments of homologous protein sequences or the physicochemical properties of amino acids in proteins ${ }^{7}$ ) and so a combination of programs should give a more confident prediction. We also analyzed the sequences using a coiled-coil predictor ${ }^{8}$ (green $=$ window of 14 residues; blue = window of 21 residues; red = window of 28 residues), which we validated against the known coiledcoil region of a Class $1 \mathrm{~N}$-terminal DDs ${ }^{9,10}$ (page 29). (Note: coiled-coil regions are predicted to be disordered by PONDR, as the monomers only fold correctly in the presence of partner). Among these programs, IUPred/ANCHOR and PONDR yielded prediction data in greatest agreement with the observed behavior of the Vir docking domains. Both programs correctly predicted that the overall VirA ${ }^{C} D D$ interaction region (residues Q6936-L6960) is largely structured, while ANCHOR identified the two $\alpha$-helices as binding motifs (data provided on page 2). Furthermore, no coiled-coil was predicted for the sequence. In the case of VirFG ${ }^{N} D D$ (residues A3-A25), both IUPred/ANCHOR and PONDR predicted a sequence on the borderline between structured and disordered, while ANCHOR identified the whole sequence as a binding region (page 2). Again, the coiled-coil prediction was negative. Gratifyingly, analogous results were obtained for the regions corresponding to VirA ${ }^{C} D D$ and VirFG ${ }^{N} D D$ from the homologous pristiniamycin PKS (SnaE2 ${ }^{C} D D$ amd SnaE3 ${ }^{N} D D$, respectively (page 3)), despite overall low levels of sequence identity between the docking regions (53\% between VirA ${ }^{C} D D$ and SnaE2 ${ }^{C} D D$ and $39 \%$ between VirFG ${ }^{\mathrm{D}} \mathrm{DD}$ and SnaE3 ${ }^{\mathrm{N}} \mathrm{DD}^{11}$ ).

We next extended this analysis to the remaining 25 putative VirA ${ }^{C} D D /$ VirFG ${ }^{N} D D$-type docking interfaces from trans-AT PKSs assigned on the basis of sequence analysis and secondary structure prediction (data provided on pages 5-31, with the data for each matched pair of docking domains shown on a single page). For 10 of these pairs (indicated in pink in the table below), the predictions were in good to excellent agreement with those for VirA ${ }^{C} D D$ and VirFG ${ }^{N} D D$ (analysis of each pair relative to VirA ${ }^{C} D D$ and VirFG ${ }^{N} D D$ is presented on each and highlighted in pink), while for 3 others, only the N-terminal docking domain shares predicted properties with VirFG ${ }^{\mathrm{N}} \mathrm{DD}$. Another important finding for 9 interfaces was the indication that the $\mathrm{N}$-terminal partner forms a coiled-coil; this implies the existence of another structural class of docking domains within trans-AT PKS (in fact, the majority of these pairs show convincing homology to the Class 2 docking domains of cis-AT PKS (see Figure 1, page 31)).

Couples analyzed:

\begin{tabular}{cccc}
\hline $\begin{array}{c}\text { C-terminal docking } \\
\text { domain }\end{array}$ & $\begin{array}{c}\text { N-terminal docking } \\
\text { domain }\end{array}$ & $\begin{array}{c}\text { C-terminal docking } \\
\text { domain }\end{array}$ & $\begin{array}{c}\text { N-terminal docking } \\
\text { domain }\end{array}$ \\
\hline VirA & VirFG & KirAV & KirAVI (coiled coil?) \\
SnaE2 & SnaE3 & MlnD & MlnE \\
AtcE & AtcF & OzmN & OzmH \\
BaeM & BaeN (coiled-coil?) & OzmH & OzmJ \\
Bat 1 & Bat2 & OzmK & OzmL \\
BryA & BryB (coiled-coil?) & RhiC & RhiD (coiled coil?) \\
BryB & BryC (coiled-coil?) & RizC & RizD (coiled-coil?) \\
ChiB & ChiC & Ta-1 & TaO (coiled-coil?) \\
ChiC & ChiD & TaiD & TaiE \\
ChiE & ChiF & TaiE & TaiK \\
DisB & DisC & Tail & TaiM \\
ElaK & ElaO & 5923 & 5922 (coiled-coil?) \\
EtnH & Etnl (coiled-coil?) & 5925 & 5924 \\
KirAll & KirAlll & & \\
\hline
\end{tabular}


Key:

Red $=$ For VirA ${ }^{C} D D /$ VirFG ${ }^{N}$ DD observed $\alpha$-helices in the complex; for all others, $\alpha$-helices predicted by PsiPred

Blue = start/end site of expression constructs for VirA ${ }^{C} D D /$ VirFG ${ }^{N} D D$, respectively All sequences are numbered starting from 1 to match the output of the programs. Note, some $\mathrm{N}$ terminal DDs were analyzed with the first $M$ present, while others were not - in the latter cases, the missing $M$ is indicated as (M).

\section{$\operatorname{Vir}{ }^{C} D D$}
29
40
46
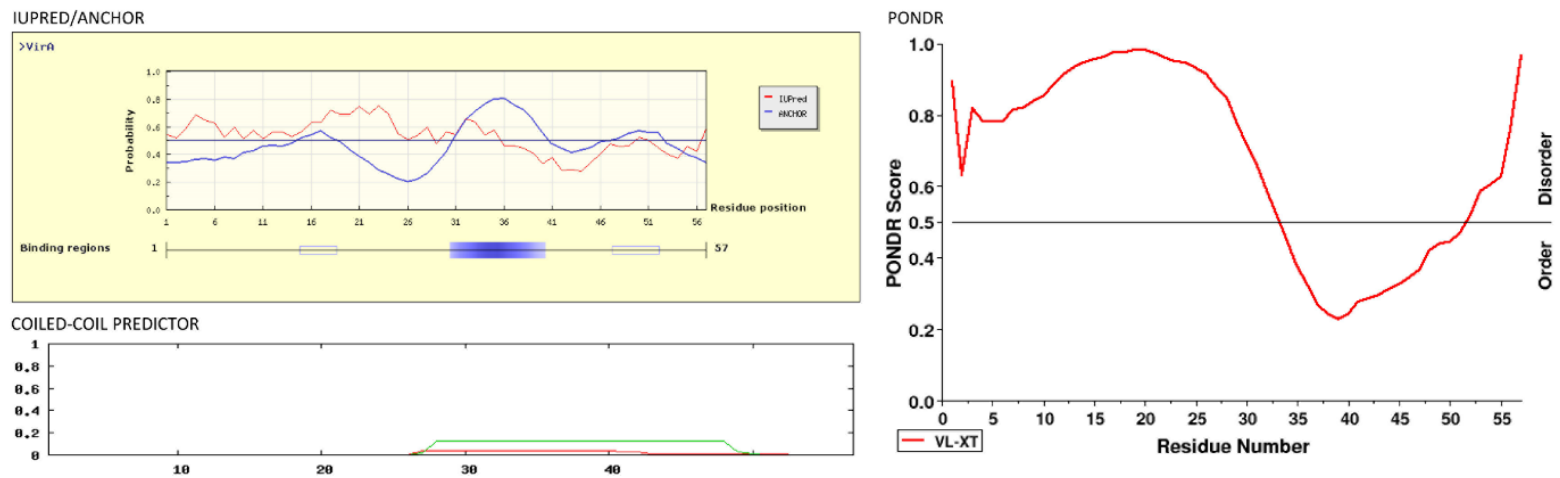

\section{VirFG ${ }^{N} D D$}

$3 \quad 11 \quad 15 \quad 25$

MDAKEILTRFKDGGLDRAAAQALLAGRTPAAAPRPSEPAAAPTRPAVPAVPAVEPAAGTTVAAEGTAG $\mathrm{RP}$

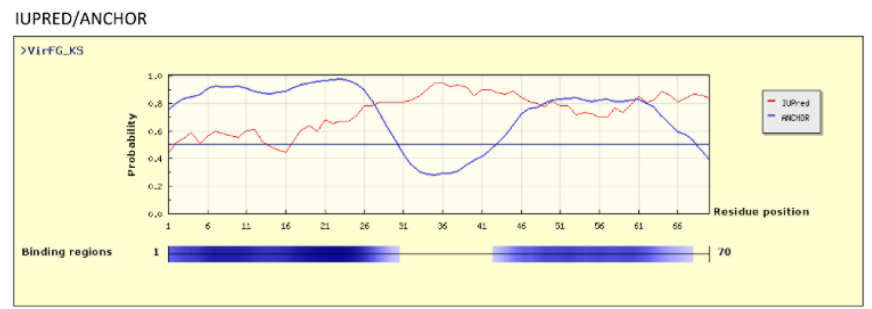

COILED-COIL PREDICTOR
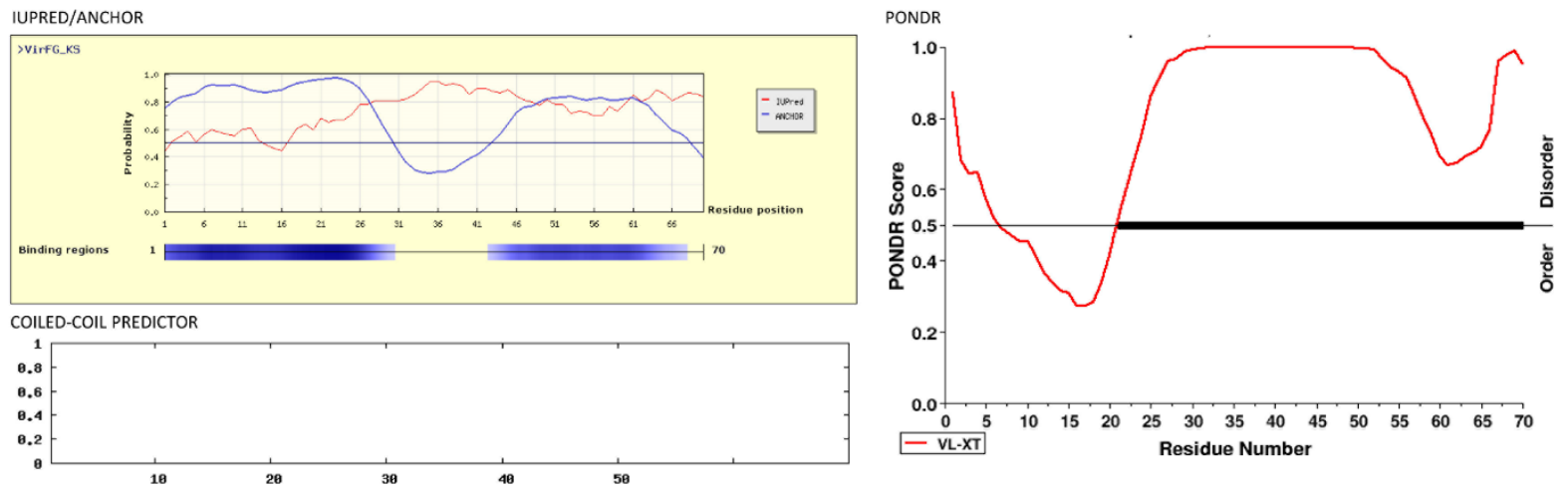


\section{SnaE2 ${ }^{\mathrm{C} D D}$}

18

30

41

TSRTAPQPAGAVPAGSVSAADLDALLAAVRDNRLTVEQALALLPQHT
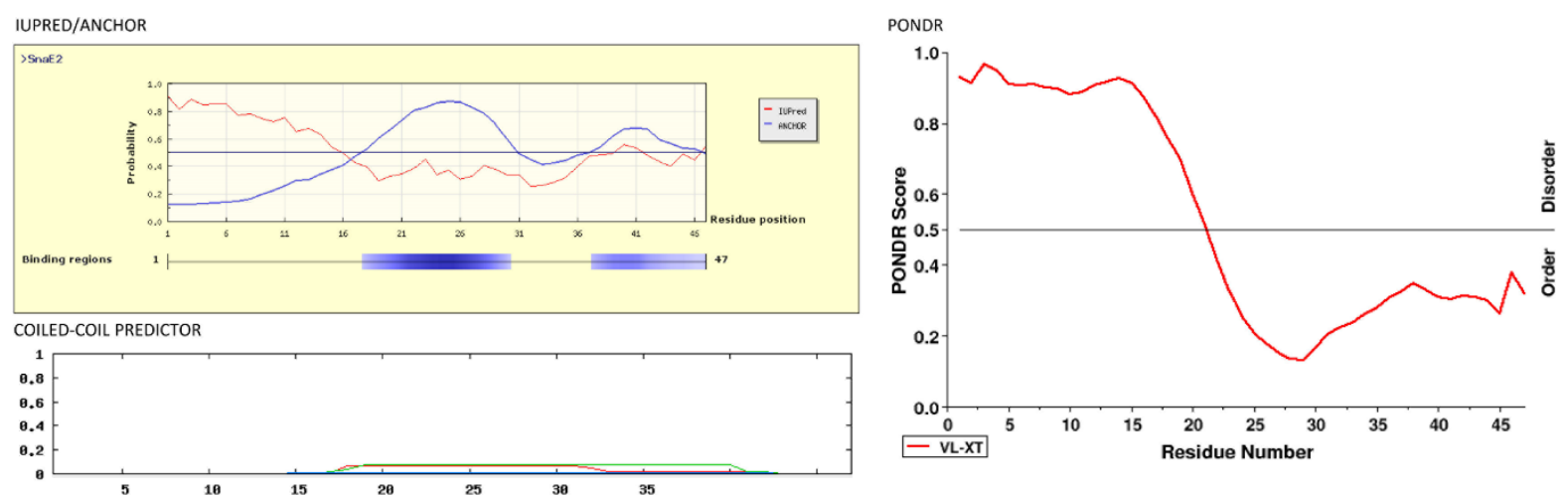

\section{OBSERVATIONS}

Predicted interaction regions (ANCHOR) overlap well with predicted $\alpha$-helices. The docking region is not predicted either to be disordered (IUPred/PONDR) or to form a coiled-coil.

Strong agreement with VirA ${ }^{\mathrm{D}} \mathrm{DD}$

\section{SnaE3 NDD}

7

44

55

MPAEPDAVDRDRAAALLRAVAGQRGPAAGAATASARPAPGGVDRAEAAALLRAAAGERRPGGDGPAGG SMGRTLPAEAGSAGRVL
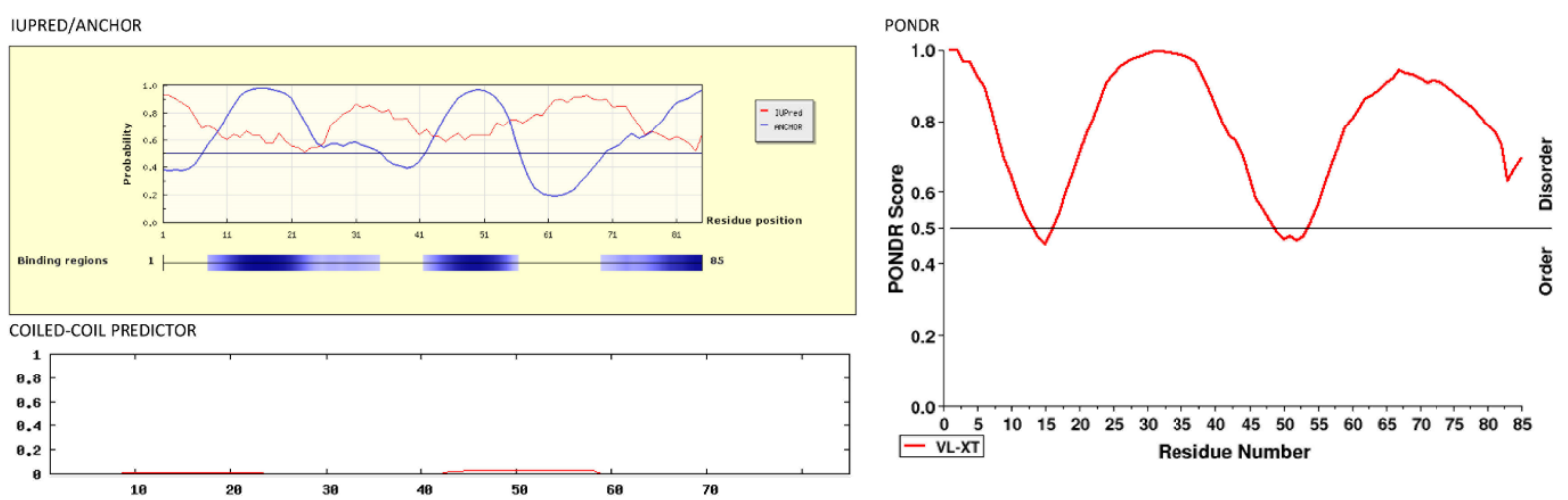

\section{OBSERVATIONS}

Predicted interaction regions (ANCHOR) overlap well with predicted $\alpha$-helices. The docking region is predicted to be largely disordered (IUPred/PONDR) but not to form a coiled-coil.

Strong agreement with VirFG ${ }^{N} D D$ 


\section{AtcE CDD}

$22 \quad 30 \quad 36 \quad 42$

HAGHAAAP PGADGAGPERATDVEDMLRRLAAGELTVAEAYTNLGGPA

IUPRED/ANCHOR

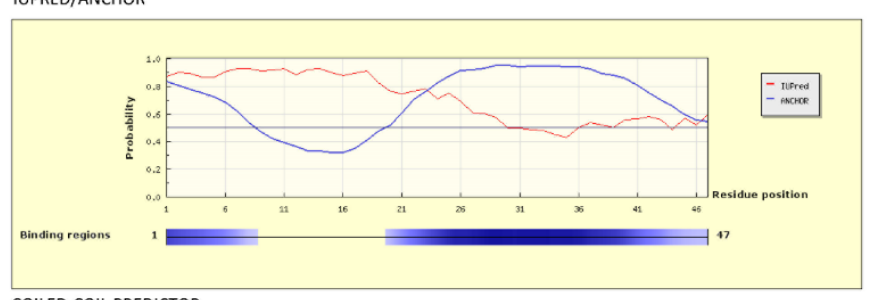

COILED-COIL PREDICTOR

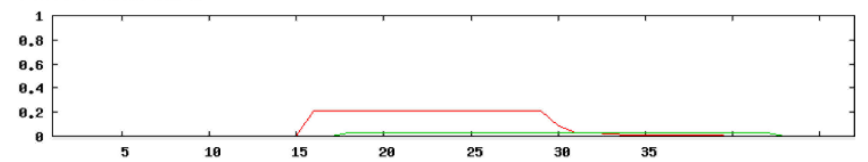

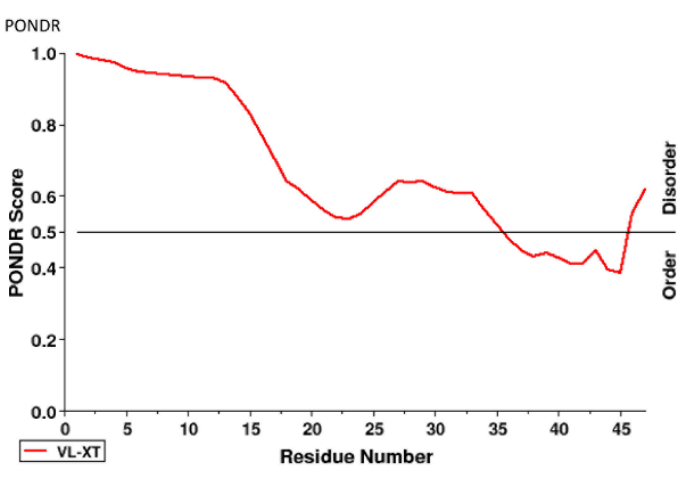

\section{OBSERVATIONS}

Predicted interaction region (ANCHOR) overlaps well with predicted $\alpha$-helices. The docking region is predicted to be borderline disordered (IUPred/PONDR) and not to form a coiled-coil.

Agreement with VirA ${ }^{\mathrm{D}} \mathrm{DD}$

\section{AtcF ${ }^{\mathrm{N} D D}$}

IUPRED/ANCHOR

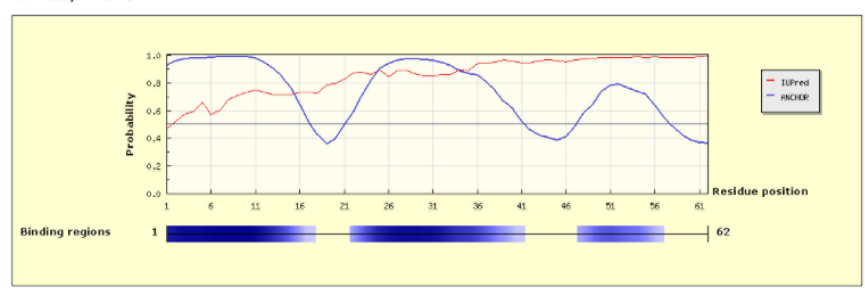

COILED-COIL PREDICTOR

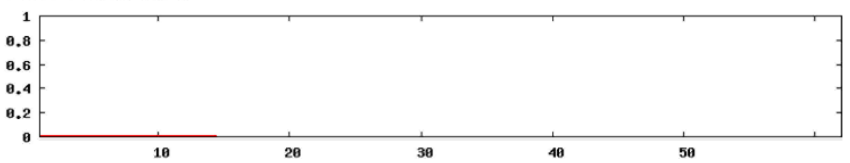

PONDR

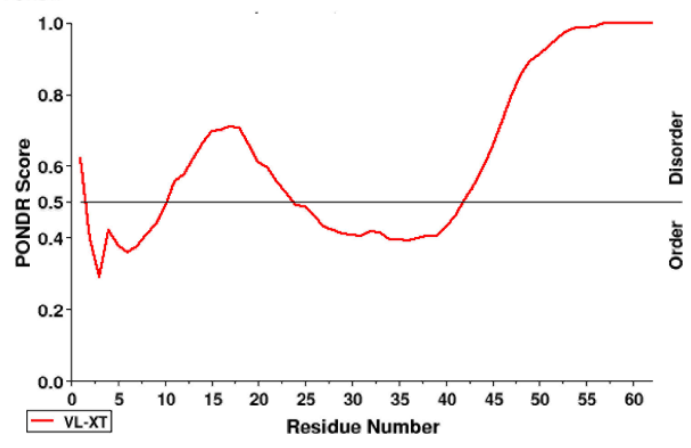

\section{OBSERVATIONS}

Predicted interaction regions (ANCHOR) overlap well with predicted $\alpha$-helices. The docking region is predicted to be largely disordered (IUPred/PONDR), but not to form a coiled-coil.

Agreement with VirFG ${ }^{\mathrm{N}} \mathrm{DD}$ 


\section{BaeM ${ }^{C} D D$}

31

IUPRED/ANCHOR

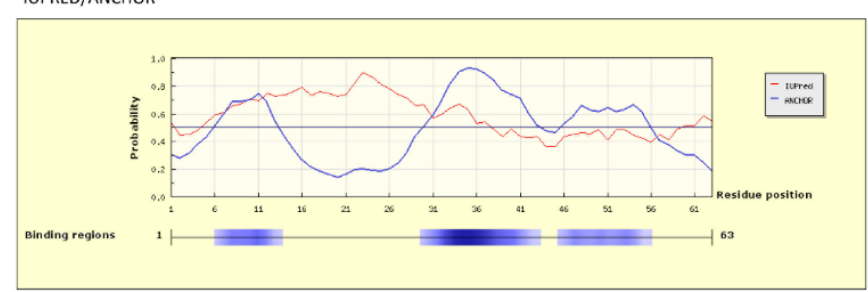

COILED-COIL PREDICTOR

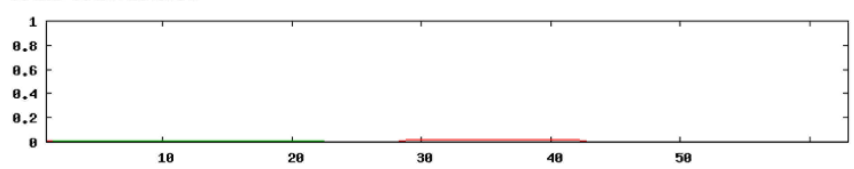

PONDR

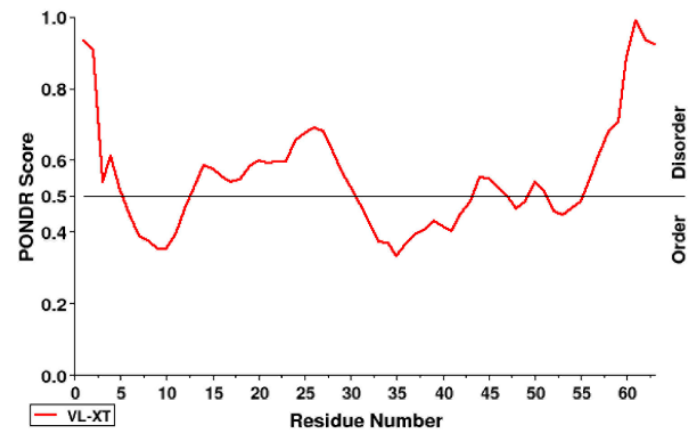

\section{OBSERVATIONS}

Predicted interaction regions (ANCHOR) overlap well with the predicted $\alpha$-helices. The docking region is predicted to be borderline disordered (IUPred/PONDR), and not to form a coiled-coil. Agreement with VirA ${ }^{\mathrm{C} D D}$

\section{BaeN NDD}

2

10

15

(M) NHKELLDAYRSGTLTIAEVEQKLQAFKRTTAKRP

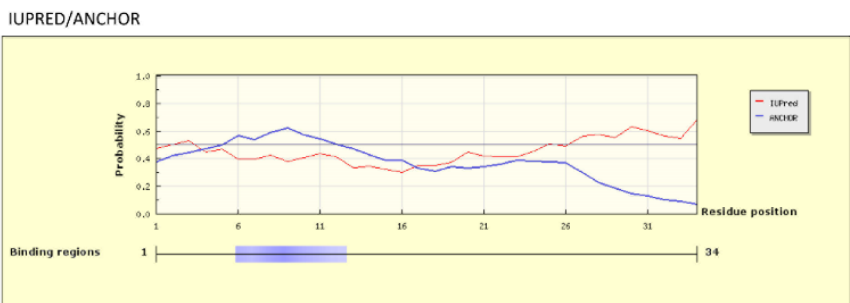

COILED-COIL PREDICTOR

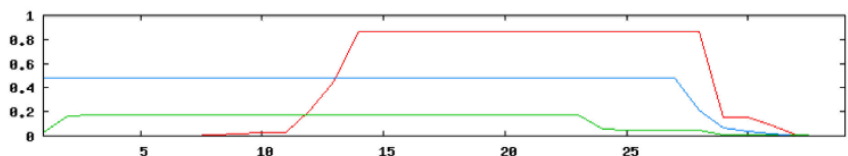

OBSERVATIONS

The first predicted $\alpha$-helix is predicted to be an interaction region (ANCHOR). The docking region is predicted to be borderline disordered (IUPred/PONDR), but to form a coiled-coil.

Does not agree with VirFG NDD; may be an alternative type of docking domain? 


\section{Bat1 ${ }^{C} D D$}

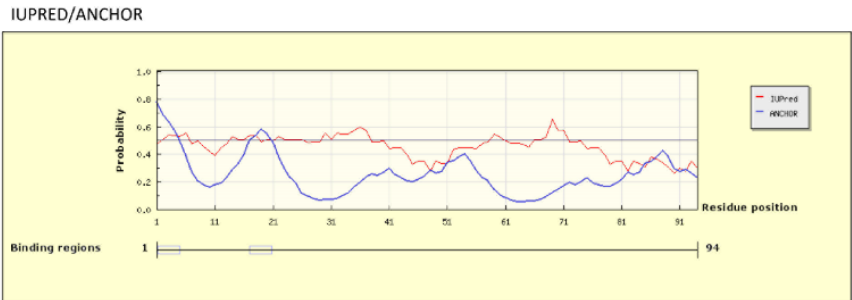

COILED-COIL PREDICTOR

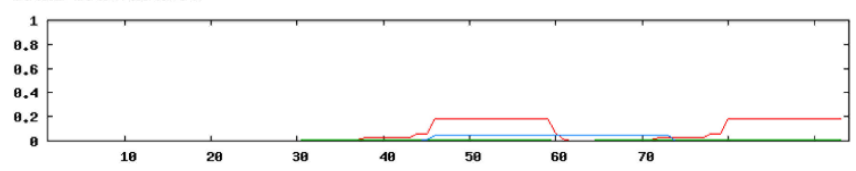

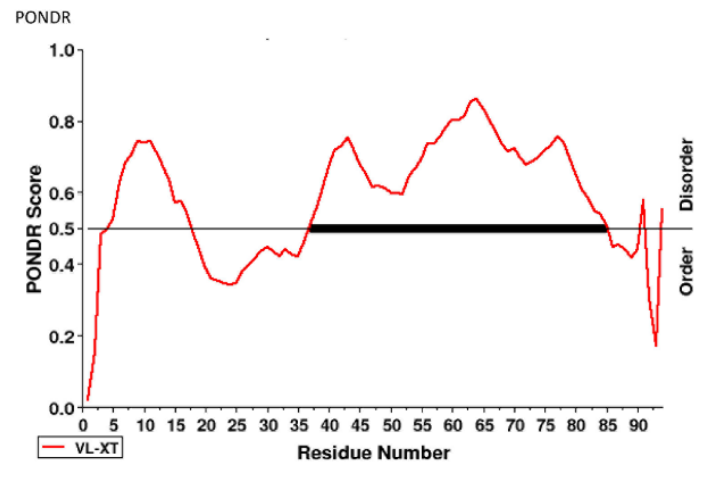

\section{OBSERVATIONS}

The predicted $\alpha$-helices are not predicted to be interaction regions (ANCHOR). The docking region is predicted to be disordered (IUPred/PONDR), and not to form a coiled-coil.

Poor agreement with VirA ${ }^{\mathrm{C} D D}$

\section{Bat2 NDD}

\section{(M) TRRDLLFAYKNRSLTTQELVAALTELNTERRFS}

IUPRED/ANCHOR

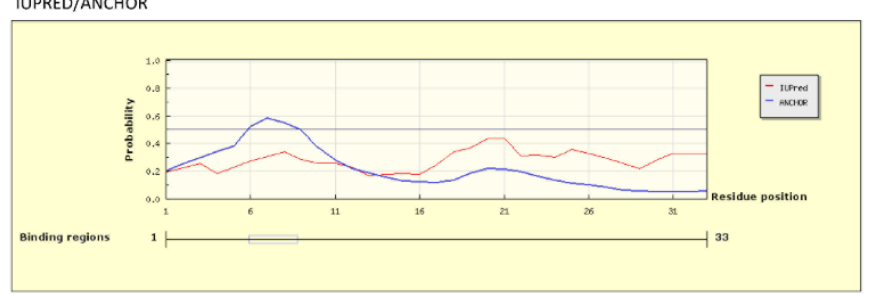

COILED-COIL PREDICTOR

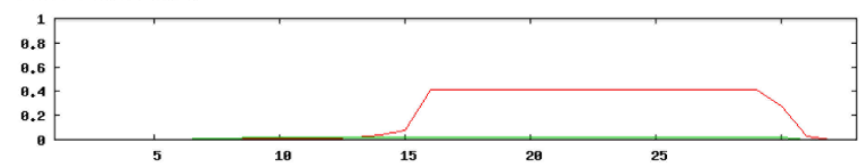

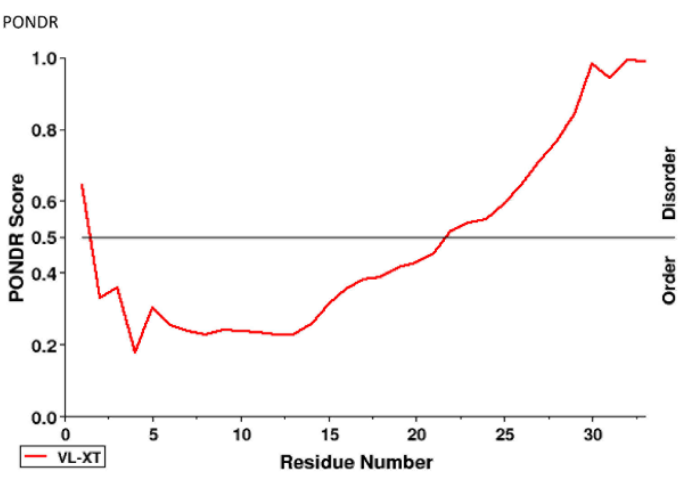

\section{OBSERVATIONS}

The secondary structure elements are not predicted to be interaction regions (ANCHOR). The docking region is not predicted to be disordered (IUPred/PONDR), and not strongly predicted to form a coiled-coil.

Poor agreement with VirFG ${ }^{\mathrm{N}} \mathrm{DD}$ 
IUPRED/ANCHOR

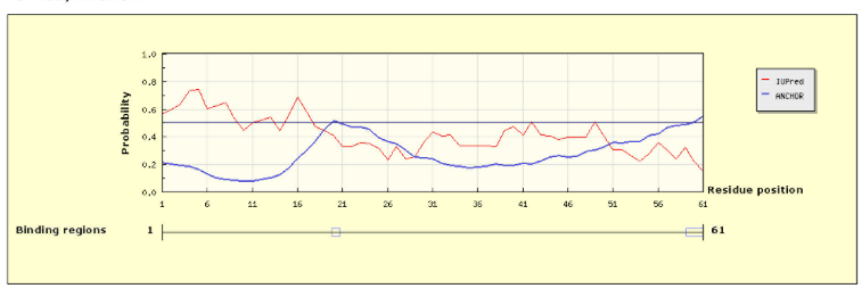

COILED-COIL PREDICTOR

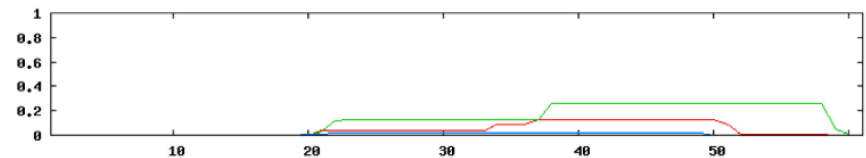

PONDR

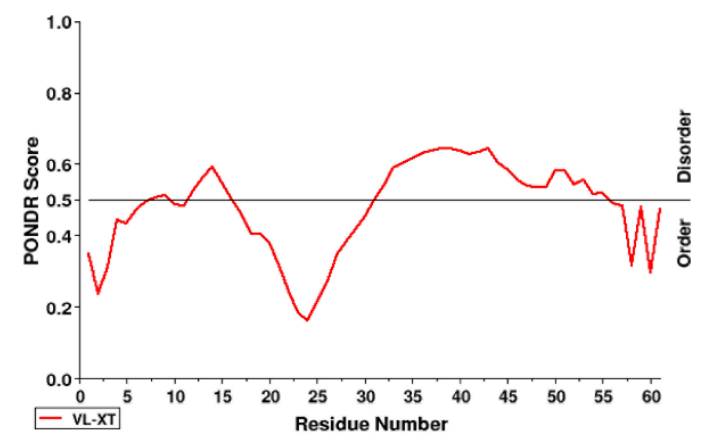

\section{OBSERVATIONS}

The predicted $\alpha$-helices are not predicted to be an interaction region (ANCHOR). The docking region is predicted to be borderline (IUPred) to mixed ordered/disordered (PONDR), and not to form a coiled-coil.

Poor agreement with VirA ${ }^{\mathrm{C} D D}$

\section{BryB NDD}

19

(M) VVVEEFFVSYRDILKALQDEKISFEEAKYKLIKRKDKKSKQRLNHDRELNRSMKITPKIVNN

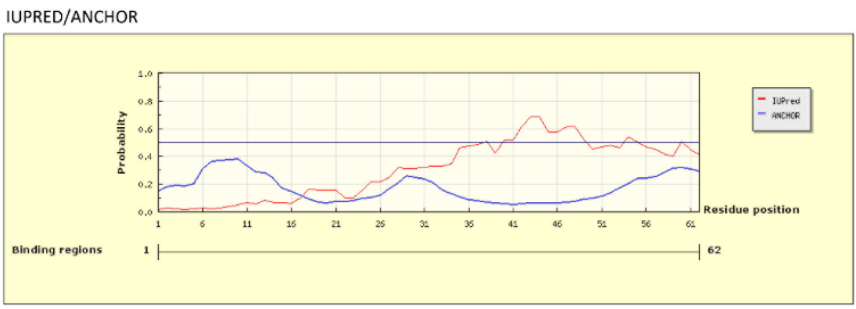

COILED-COIL PREDICTOR

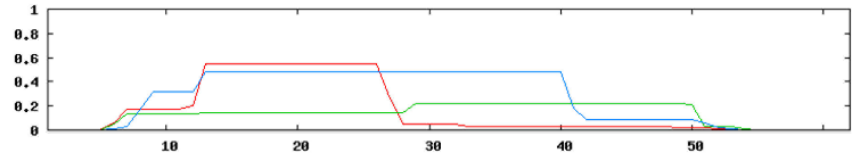

\section{OBSERVATIONS}

The predicted $\alpha$-helices are not predicted to be interaction regions (ANCHOR). The first $\alpha$-helix is predicted to be ordered and the second disordered (IUPred/PONDR), and the overall domain to form a coiled-coil.

Does not agree with VirFG NDD; may be an alternative type of docking domain? 


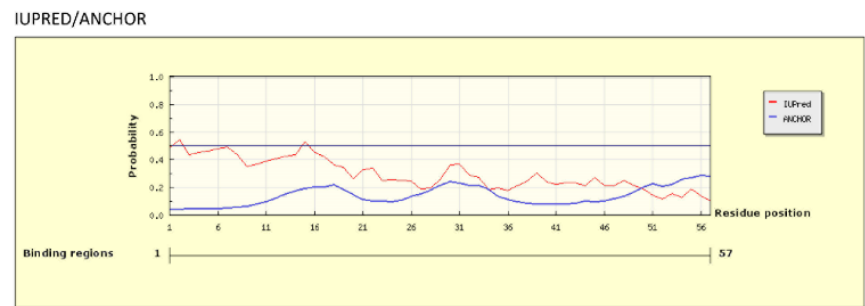

COILED-COIL PREDICTOR

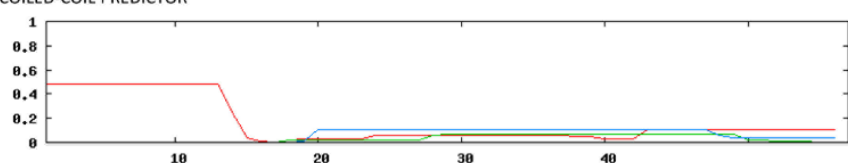

PONDR

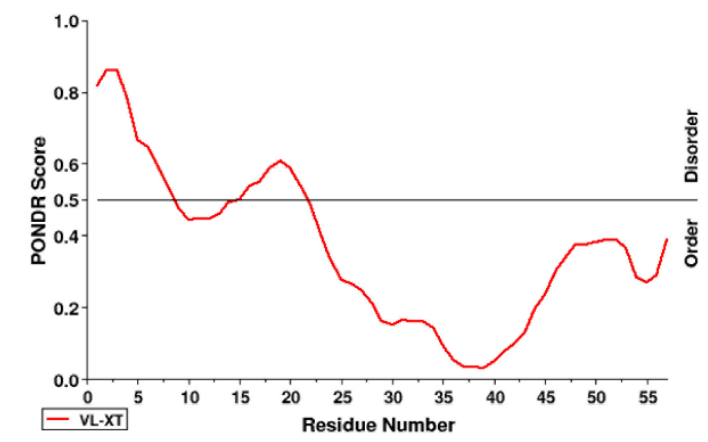

\section{OBSERVATIONS}

The predicted $\alpha$-helices are not predicted to be an interaction region (ANCHOR). The docking region is predicted to be ordered (IUPred/PONDR), and not to form a coiled-coil.

Poor agreement with VirA ${ }^{\mathrm{C}} \mathrm{DD}$

\section{BryC NDD}

$4 \quad 7625$

(M) KAKVIFQGLKSNEISIEQAEKMLLSLDTESKHGHPESKLSDIKSDLHDD

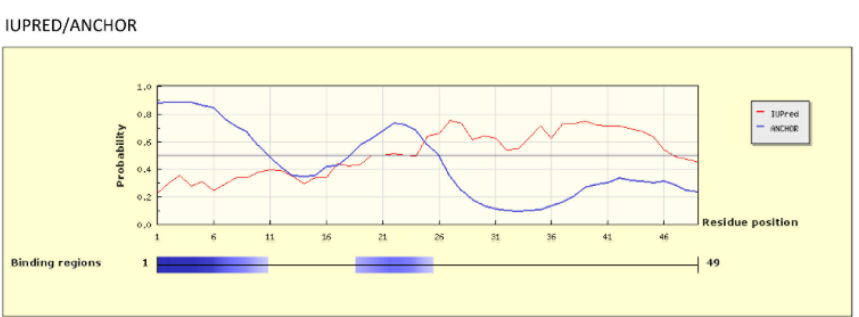

COILED-COIL PREDICTOR

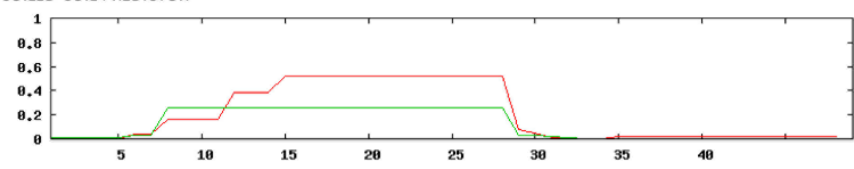

PONDR

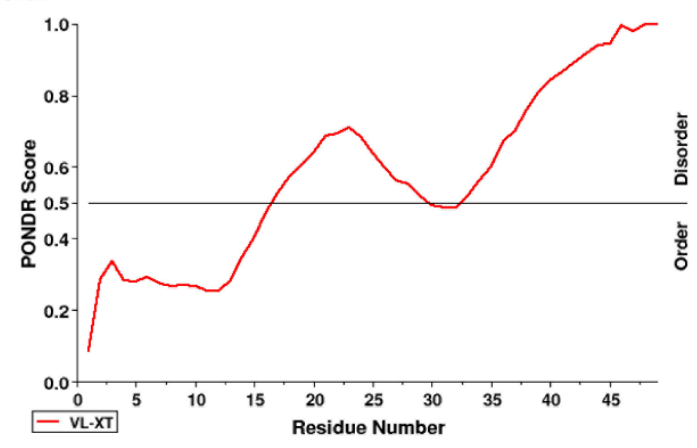

\section{OBSERVATIONS}

The predicted secondary structure elements are predicted to be interaction regions (ANCHOR). The docking region is predicted to be largely ordered (IUPred/PONDR), and there is some indication for coiled-coil formation.

Does not agree with VirFG NDD; may be an alternative type of docking domain? 


\section{ChiB ${ }^{\mathrm{C} D D}$}

70

IUPRED/ANCHOR

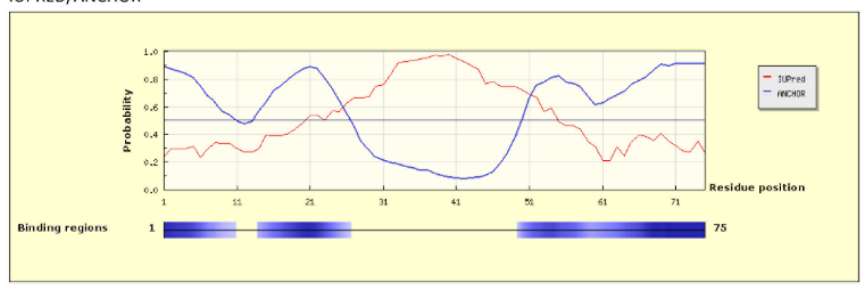

COILED-COIL PREDICTOR

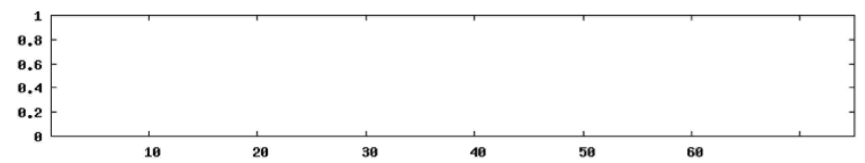

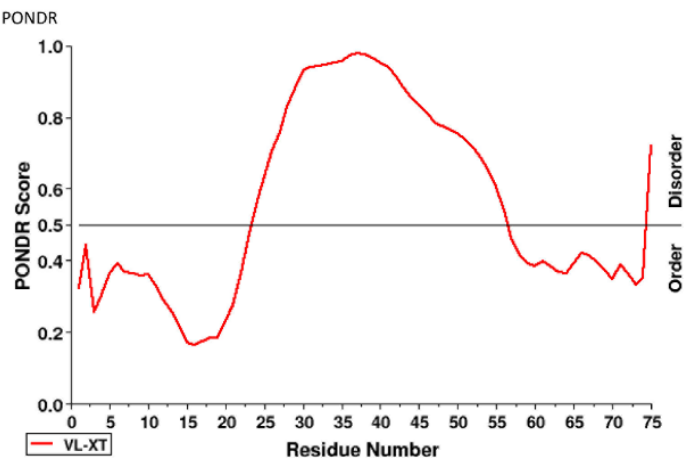

\section{OBSERVATIONS}

Predicted interaction region (ANCHOR) overlaps well with predicted $\alpha$-helices. The docking region is not predicted to be disordered (IUPred/PONDR) nor to form a coiled-coil.

\section{Strong agreement with VirA ${ }^{\mathrm{C}} \mathrm{DD}$}

\section{ChiC ${ }^{\mathrm{N} D D}$}

$4 \quad 12 \quad 16 \quad 28$

(M) KRDPRVIFEQLKNGQISRAEAHRLLKARSDGREQEATGPGAEEGAAAEARAPSAPPPQPASSDSD VG

IUPRED/ANCHOR

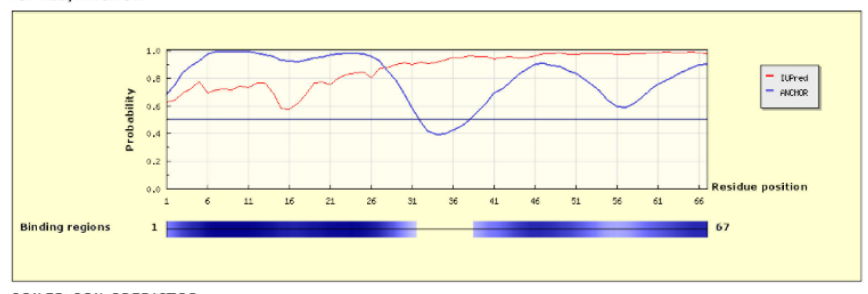

COILED-COIL PREDICTOR

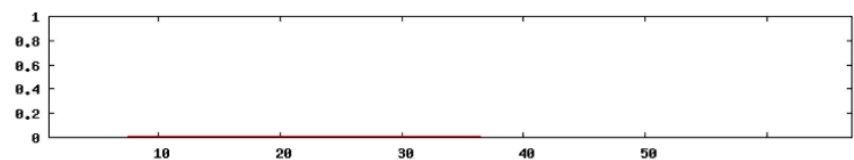

PONDR

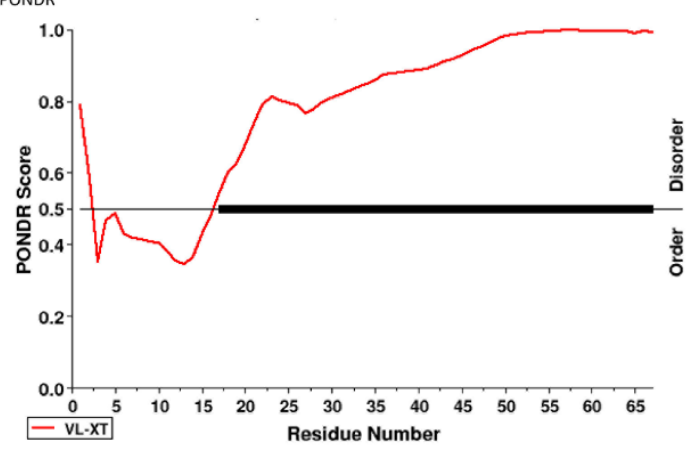

\section{OBSERVATIONS}

Predicted interaction regions (ANCHOR) overlap well with predicted $\alpha$-helices. The docking region is predicted to be borderline disordered (IUPred/PONDR), but not to form a coiled-coil.

\section{Strong agreement with VirFG ${ }^{N} D D$}




\section{Chic ${ }^{\mathrm{C} D D}$}

IUPRED/ANCHOR

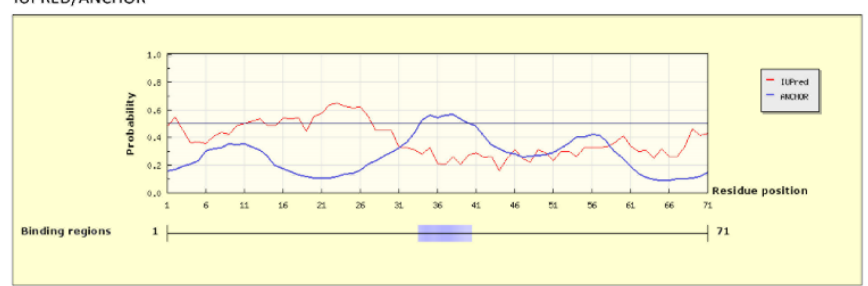

COILED-COIL PREDICTOR

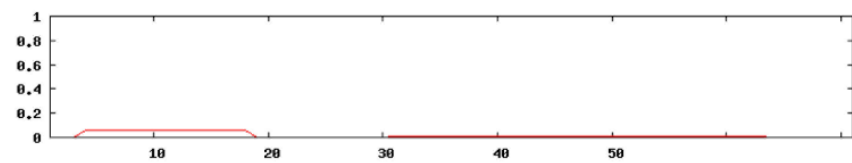

PONDR

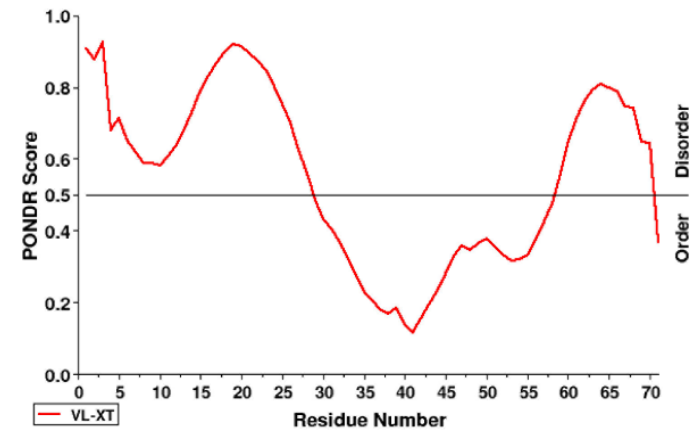

\section{OBSERVATIONS}

Predicted $\alpha$-helices not confidently predicted as interaction regions. The C-terminal extreme of the docking region is predicted to be disordered (IUPred/PONDR), while the whole region is not predicted to form a coiled-coil.

Relatively poor agreement with VirA ${ }^{\mathrm{C}} \mathrm{DD}$

\section{ChiD NDD}

\section{5}

MQRPDRGRRLYRMEKAVTDAMFDERSREEKLSVFRALRDRLRRTPPQHGGAADGAGPRAPVLAPDPQN RHRPFP

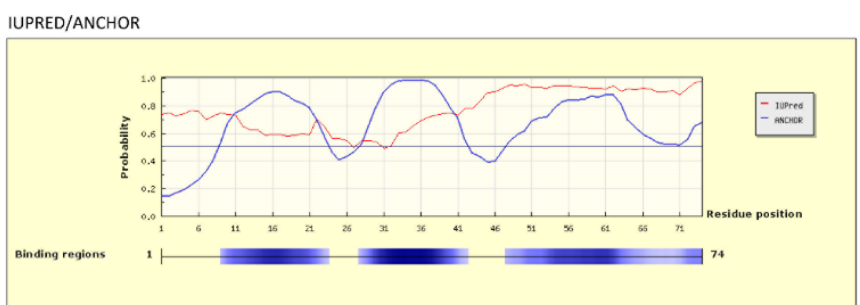

COILED-COIL PREDICTOR

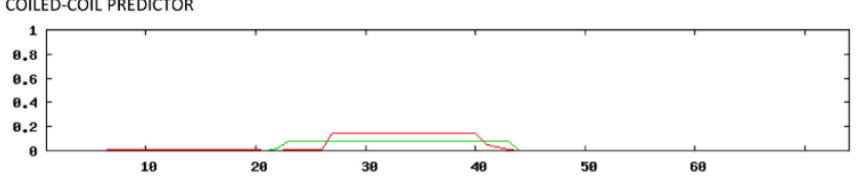

PONDR

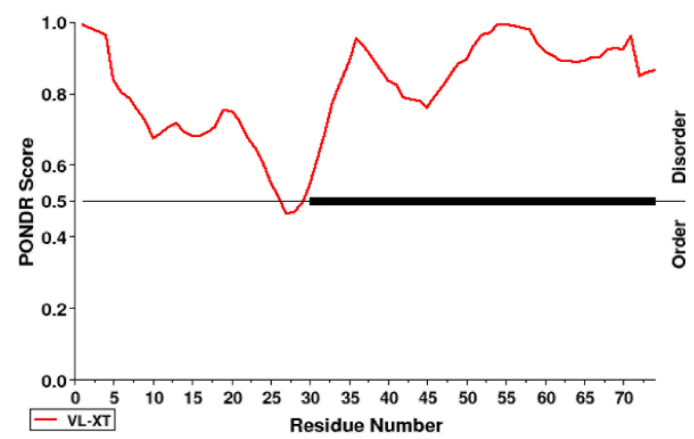

\section{OBSERVATIONS}

Predicted interaction regions (ANCHOR) overlap well with predicted $\alpha$-helix. The docking region is strongly predicted to be disordered (IUPred/PONDR), but not to form a coiled-coil.

Strong agreement with VirFG ${ }^{\mathrm{D} D D}$ 


\section{ChiE ${ }^{\mathrm{C} D D}$}

VDHHEAALRASEPPGVGGGGAEPVRPRANPERPERADPPERAGVAPGRRAPSPPAAAVERFDGAVPED LAGEVARMSDAEVERLLGWLQGGGAGGAGGAGGAGATNGSSAVRASLDDGQEREAERRRLDRGPSGEW $\mathrm{R}$

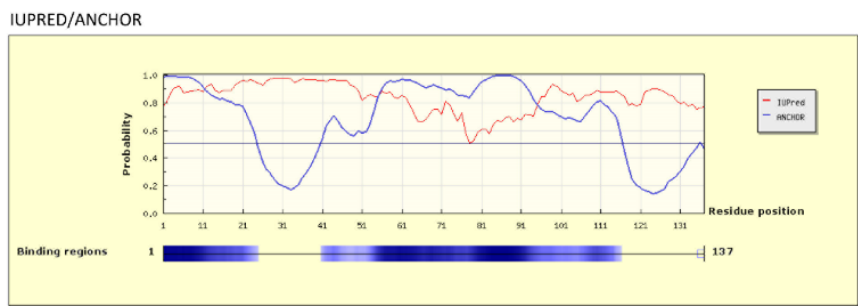

COILED-COIL PREDICTOR
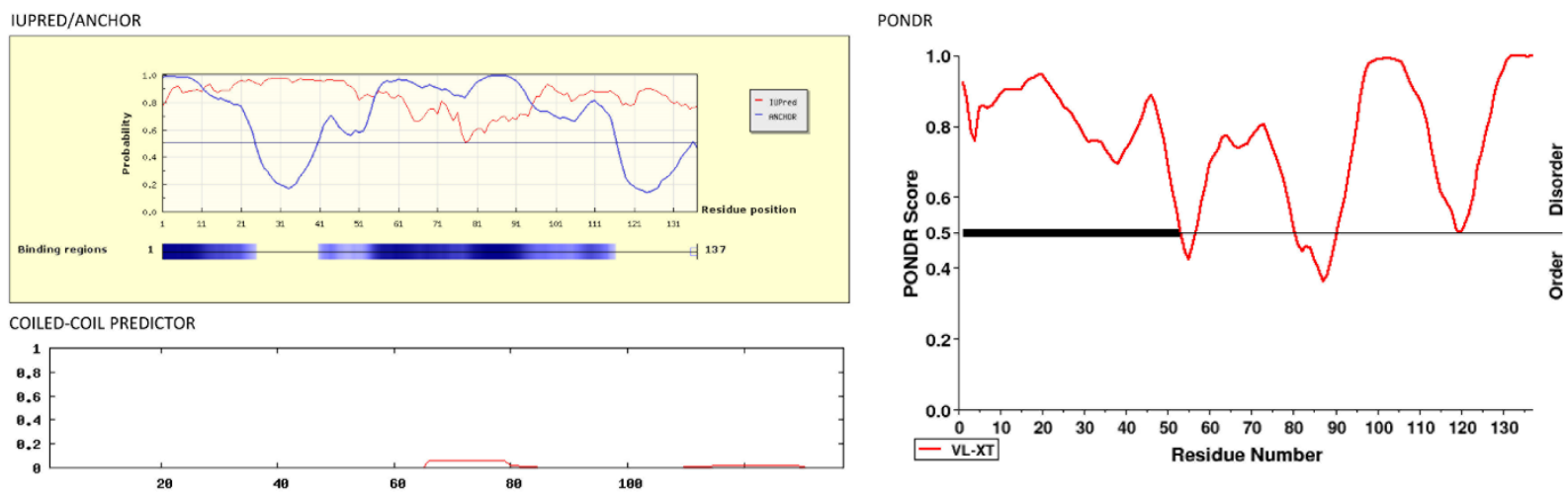

\section{OBSERVATIONS}

Predicted $\alpha$-helices predicted as interaction regions. The docking region is predicted to be disordered (IUPred/PONDR), and not predicted to form a coiled-coil.

Some agreement with VirA ${ }^{C} D D$

\section{ChiF ${ }^{N}$ DD}

5
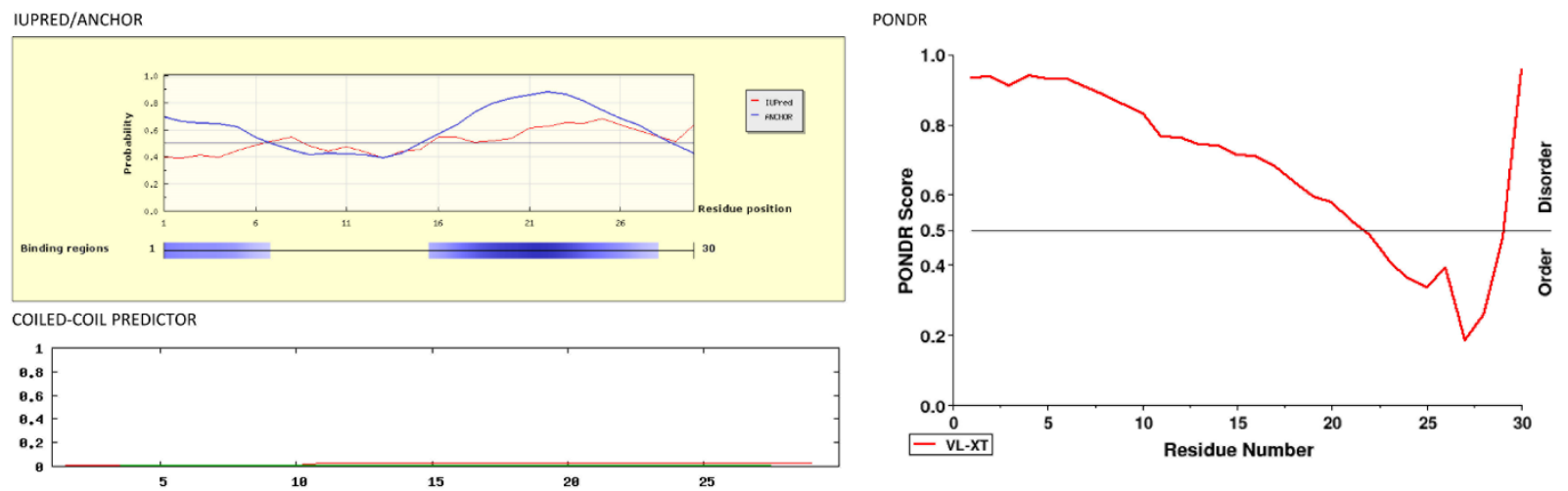

\section{OBSERVATIONS}

A portion of the predicted interaction regions (ANCHOR) overlaps well with the predicted $\alpha$-helix. The docking region is predicted to be disordered (IUPred/PONDR), but not to form a coiled-coil. Agreement with VirFG NDD 


\section{DisB ${ }^{C} D D$}

IUPRED/ANCHOR

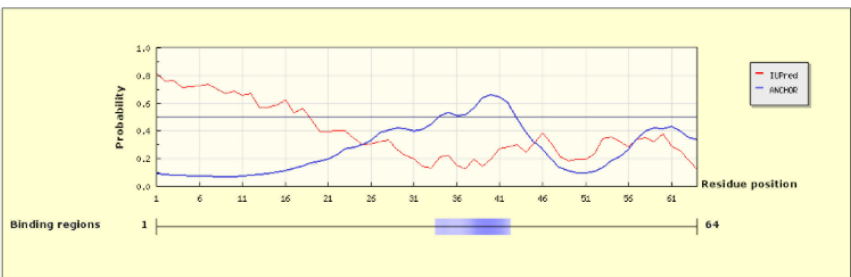

COILED-COIL PREDICTOR

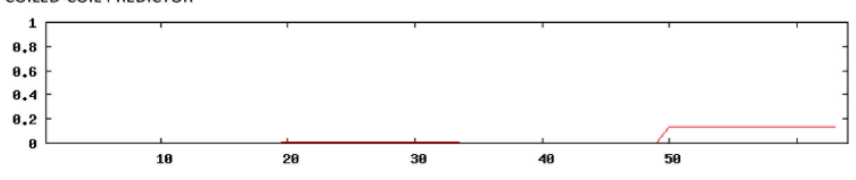

\section{OBSERVATIONS}

The first predicted $\alpha$-helix is predicted to be an interaction region (ANCHOR), though not the second. The docking region is predicted to be largely ordered (IUPred/PONDR), and not to form a coiled-coil. Agreement with VirA ${ }^{\mathrm{D} D D}$

\section{DisC NDD}

$6 \quad 11 \quad 15$

30

MESAMTIQEFANLSAEEKVQVLRLRDRRASWQAAPEGPAASAQPSLRPVITARPGDRFLPFP
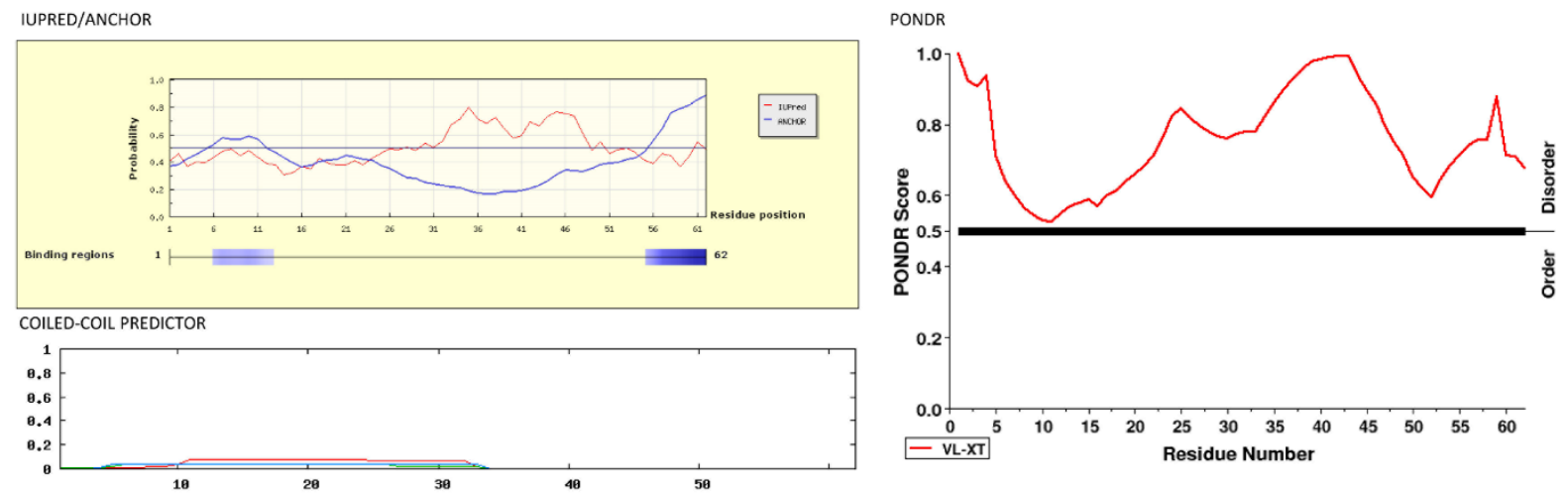

\section{OBSERVATIONS}

The first predicted $\alpha$-helix is predicted to be an interaction region (ANCHOR), though not the second. The docking region is predicted to be borderline (IUPred) to strongly disordered (PONDR), and not predicted to form a coiled-coil.

Agreement with VirFG ${ }^{\mathrm{N} D D}$ 


\section{ElaK ${ }^{\mathrm{C} D D}$}

IUPRED/ANCHOR

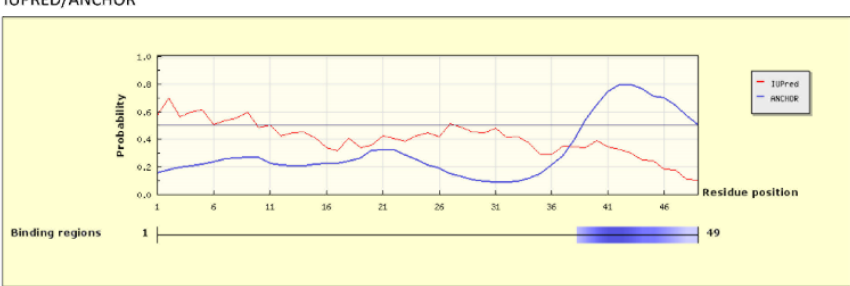

COILED-COIL PREDICTOR

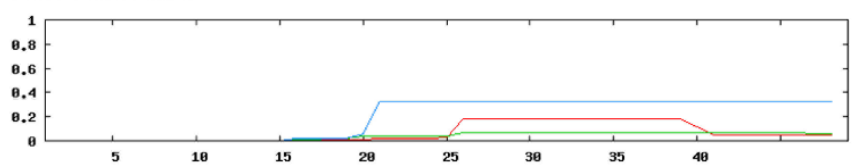

PONDR

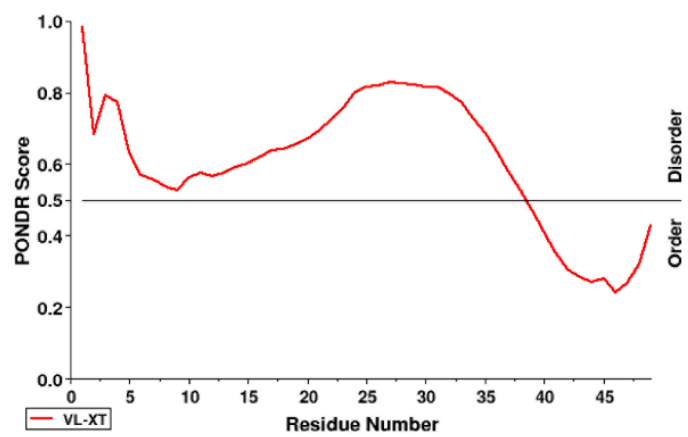

\section{OBSERVATIONS}

Second $\alpha$-helicx predicted as interaction region. The first $\alpha$-helix is predicted to be borderline to disordered while the second is ordered (IUPred/PONDR), and neither is strongly predicted to form a coiled-coil.

Some agreement with VirA ${ }^{C} D D$

\section{ElaO NDD}
2
10
17
26

(M) DSLAILKALSTGAISAGAAREALKQLAVAPVSVKPGHDARATP

IUPRED/ANCHOR

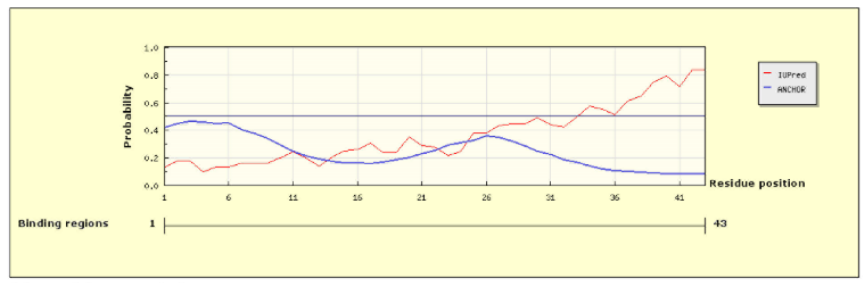

COILED-COIL PREDICTOR

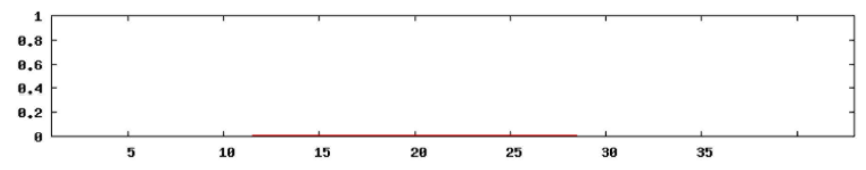

PONDR

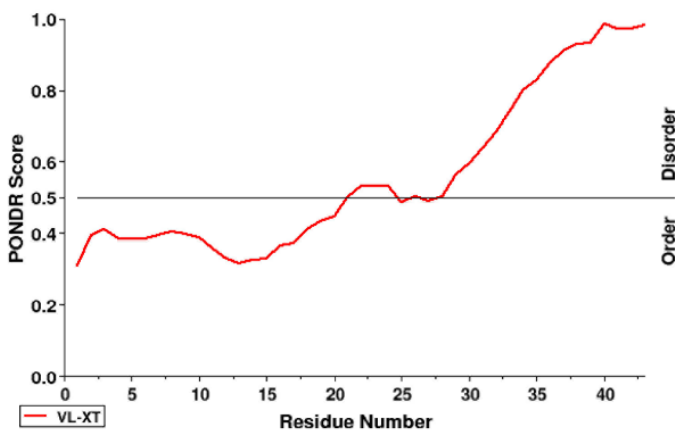

\section{OBSERVATIONS}

The predicted $\alpha$-helices are not predicted to be interaction regions (ANCHOR). The docking region is predicted to be borderline disordered (IUPred/PONDR), but not to form a coiled-coil.

Some agreement with VirFG ${ }^{\mathrm{N} D D}$ 


\section{EtnH ${ }^{C} D D$}

IUPRED/ANCHOR

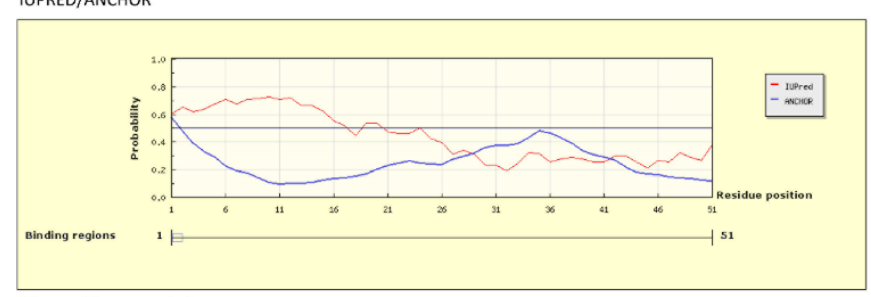

COILED-COIL PREDICTOR

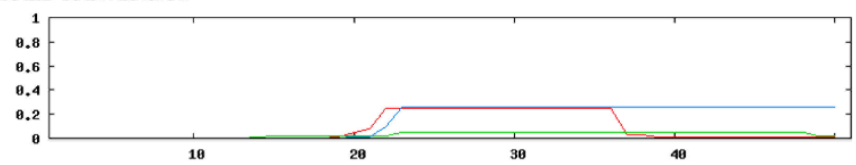

PONDR

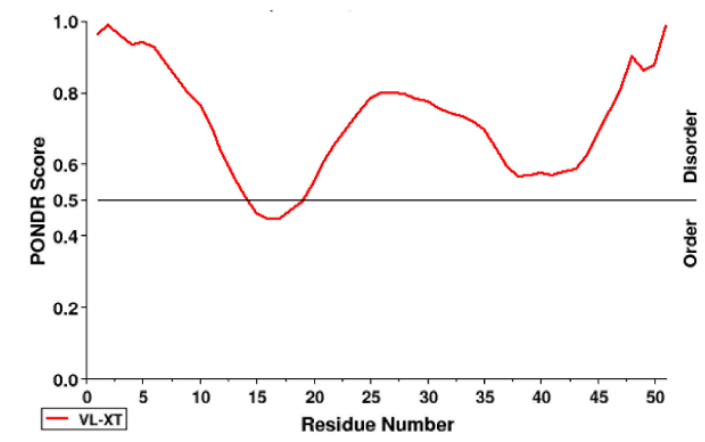

\section{OBSERVATIONS}

The predicted $\alpha$-helix is not predicted to be an interaction region (ANCHOR). The docking region is predicted to be ordered (IUPred) to disordered (PONDR), and not to form a coiled-coil.

Poor agreement with VirA ${ }^{\mathrm{C}} \mathrm{DD}$

\section{Etnl NDD}

10

(M) PEHSYEPRGESTVKSQIEALLREYQSGLLSEAELRARYAQLVASGSDGAPARPADRAGATQTVRR
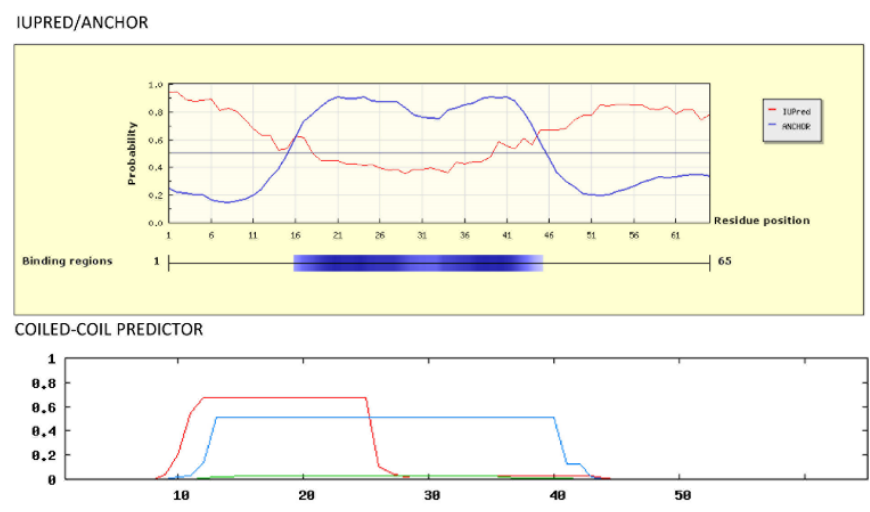

PONDR

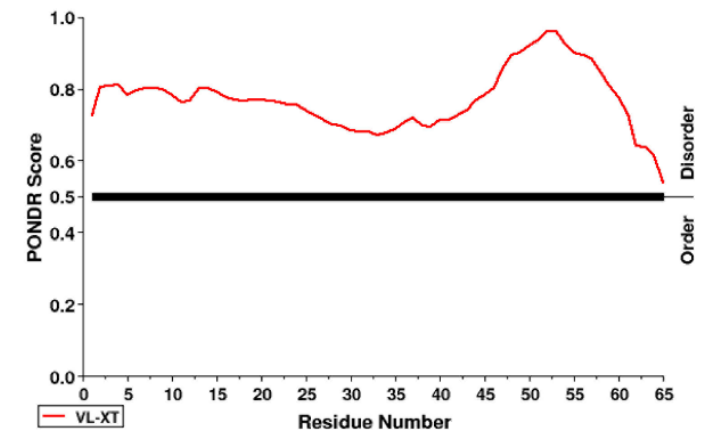

\section{OBSERVATIONS}

The predicted $\alpha$-helix is predicted to be an interaction region (ANCHOR). The docking region is predicted to be borderline (IUPred) to strongly disordered (PONDR), and there is some indication for coiled-coil formation.

Does not agree with VirFG ${ }^{N} D D$; may be an alternative type of docking domain? 


\section{KirAll ${ }^{C}$ DD}

HDKYGPLPATATPEPPPAPVTSSATAPEPTSVPVASSAAAPAPI PAPVAPSAAAPAPVASSAAAPEPP PAPVAADAVGALLAGLRDGSVTVDDALAELRQGNVR
72
85
91
99

IUPRED/ANCHOR

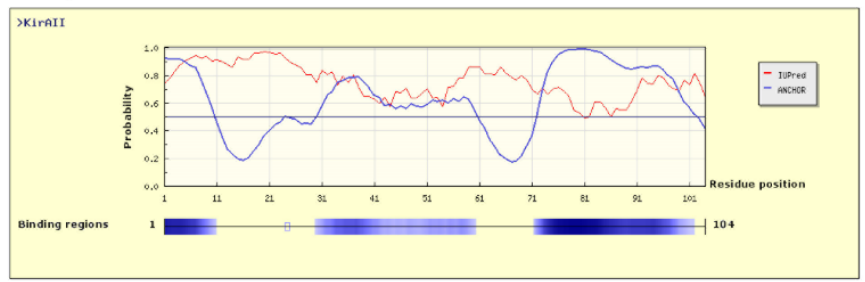

COILED-COIL PREDICTOR
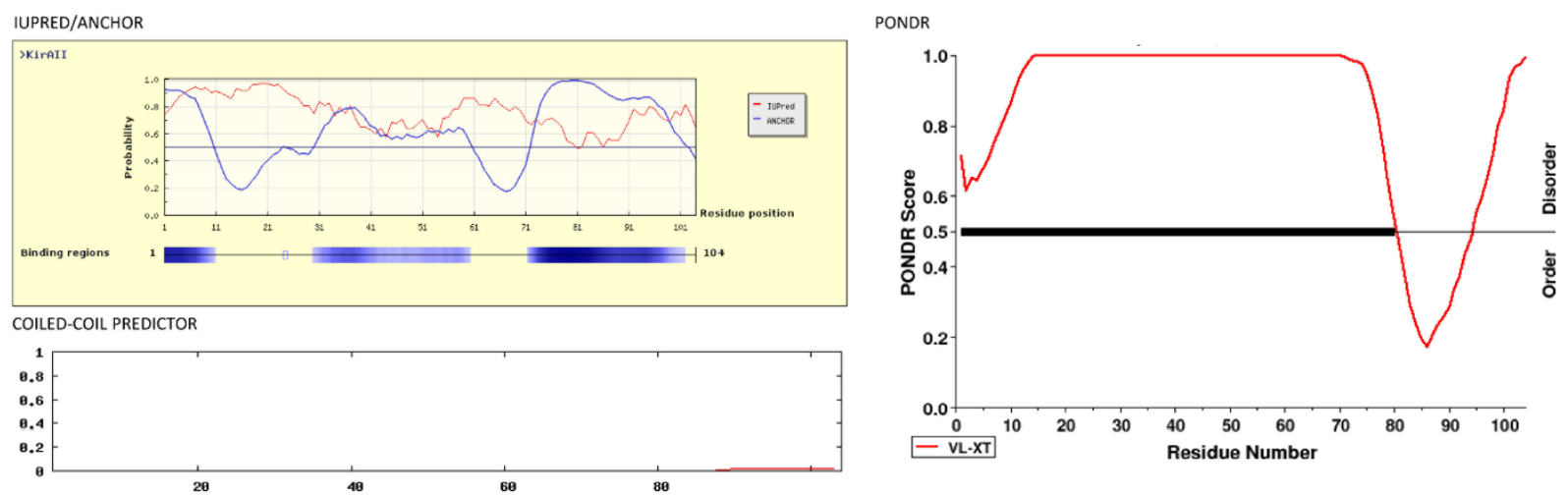

\section{OBSERVATIONS}

Predicted interaction region (ANCHOR) overlaps well with predicted $\alpha$-helices. The docking region is predicted to be mixed ordered/disordered (IUPred/PONDR), and not to form a coiled-coil.

\section{Agreement with VirA ${ }^{\mathrm{C} D D}$}

\section{KirAIII ND}
3
13
18
29

MSRALRDVLAAVAAGELPDEIAESLLRGLSDPVPEPGPYP

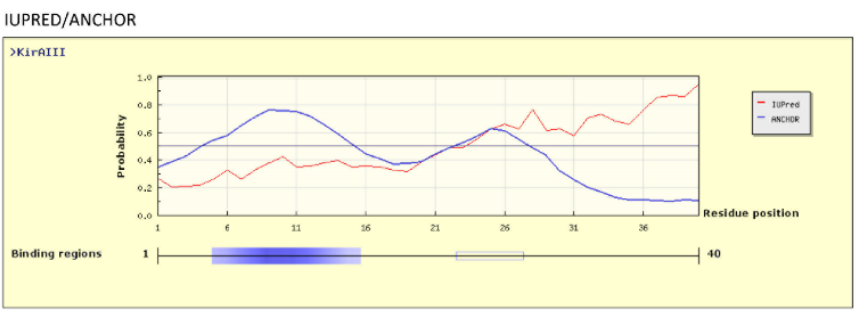

COILED-COIL PREDICTOR

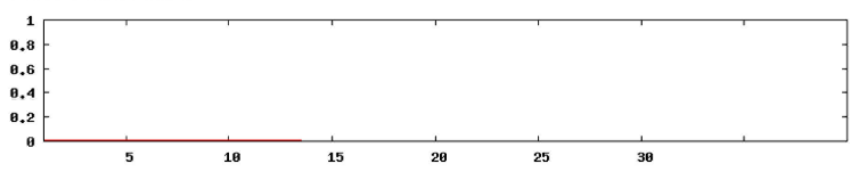

PONDR

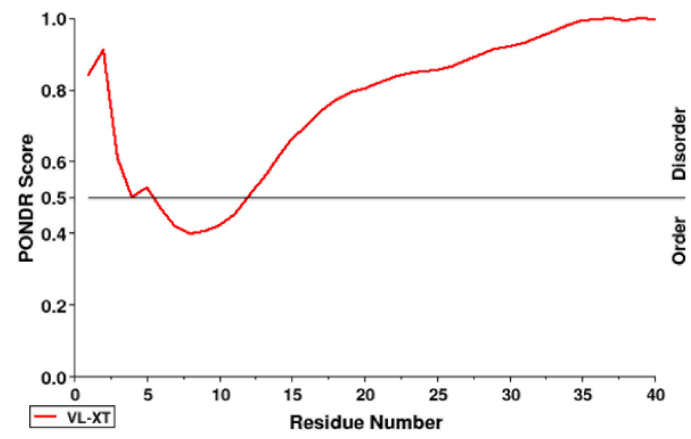

\section{OBSERVATIONS}

Predicted interaction regions (ANCHOR) overlap well with predicted $\alpha$-helices (though the second interaction region isn't strong). The docking region is predicted to be borderline disordered (IUPred/PONDR), but not to form a coiled-coil.

\section{Agreement with VirFG ${ }^{\mathrm{D} D}$}




\section{KirAV CDD}

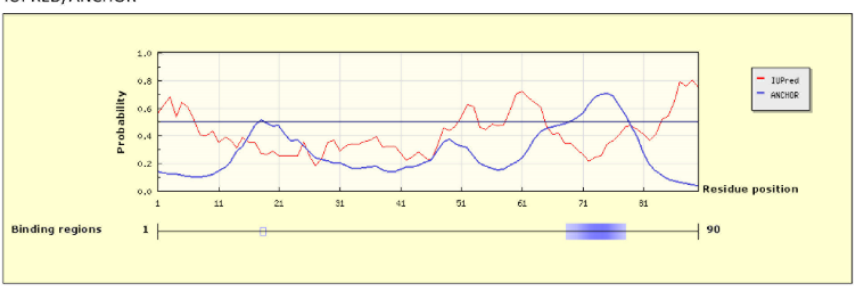

COILED-COIL PREDICTOR
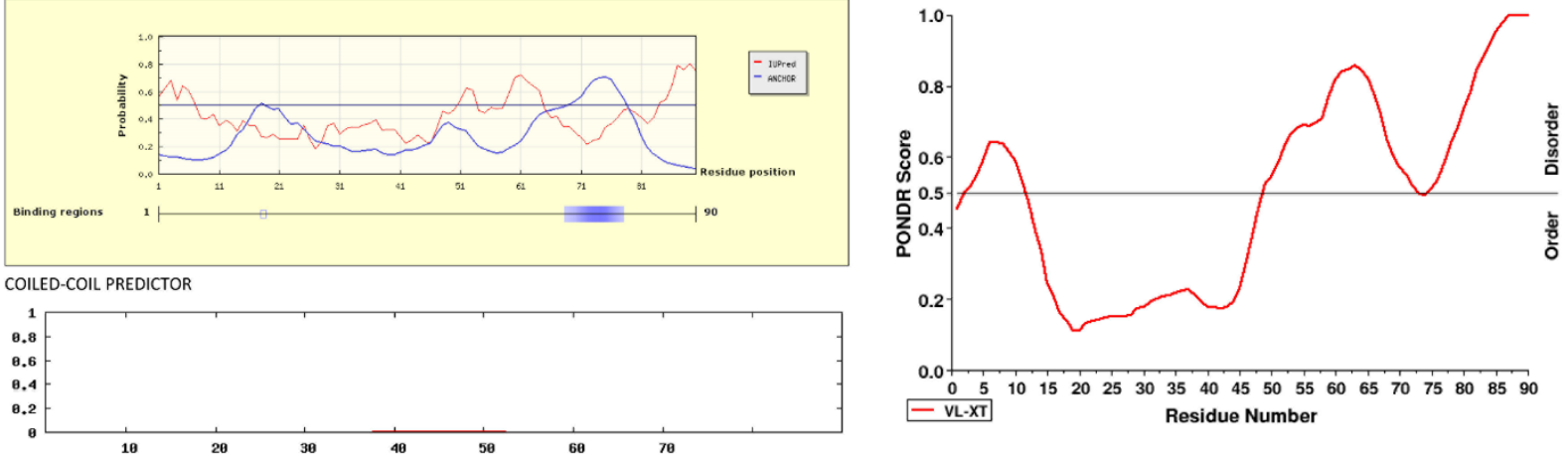

\section{OBSERVATIONS}

The second $\alpha$-helix is predicted to be an interaction region (ANCHOR). The docking region is predicted to be mixed ordered/disordered (IUPred/PONDR), and not to form a coiled-coil. Some agreement with VirA ${ }^{C} D D$

\section{KirAVI NDD}

\section{MSSEDKLRDYLRRVTAELQVTRRRLREVESRSG}

IUPRED/ANCHOR

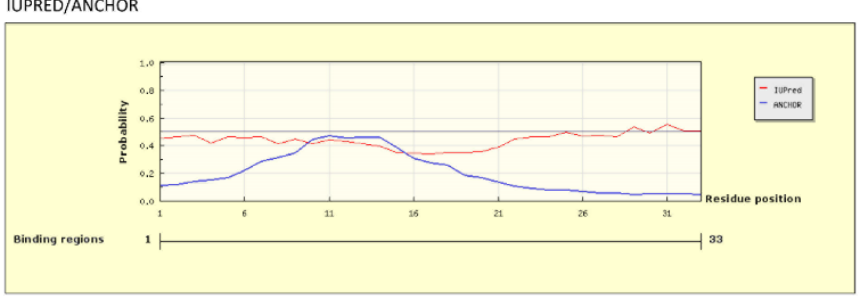

COILED-COIL PREDICTOR

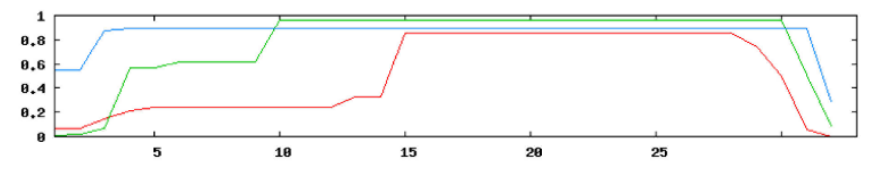

PONDR

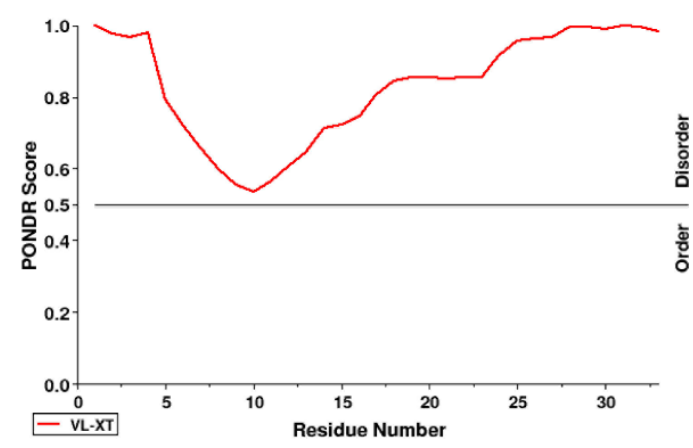

\section{OBSERVATIONS}

The predicted $\alpha$-helix is not predicted to be an interaction region. The docking region is predicted to be disordered (IUPred/PONDR) and strongly predicted to form a coiled-coil.

Does not agree with VirFG ${ }^{\mathrm{N}} \mathrm{DD}$; may be an alternative type of docking domain? 


\section{$M \ln { }^{C} D D$}

49

60

SS IRNRSADKPYNGNFREKSGFGLEEIAYTLQTGREPFECRLAITCSNIGDLLEKLNGYIQGSRLQGV YEGNVTDFDSLYAPLFGGPEGHAYVQSLIQQNKPAKLAQLWTIGCEISWEDLYEEELKPVKLSLPLYP

IUPRED/ANCHOR

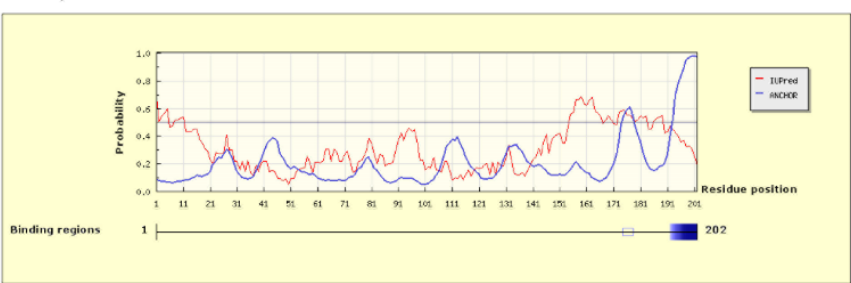

COILED-COIL PREDICTOR

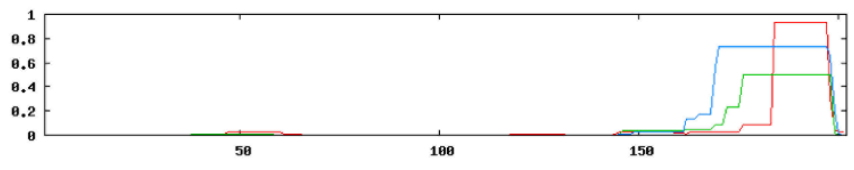

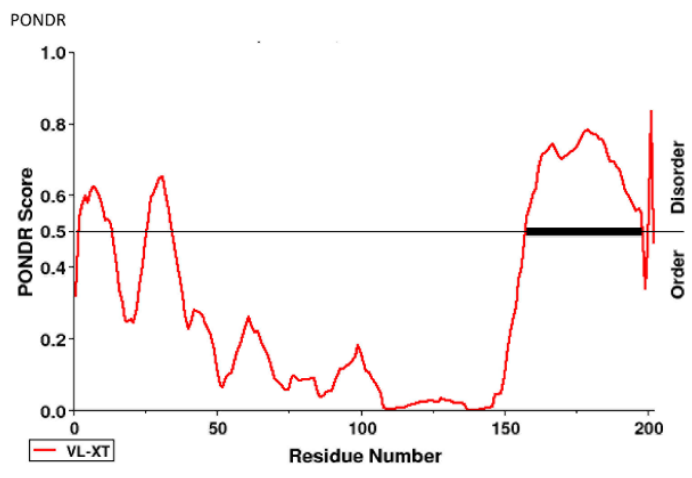

\section{OBSERVATIONS}

The predicted $\alpha$-helices are not predicted to be interaction regions (ANCHOR). The docking region is predicted to be strongly ordered (IUPred/PONDR), and not to form a coiled-coil.

Poor agreement with VirA ${ }^{C} D D$

\section{MInE NDD}

$3 \quad 16 \quad 2123$

(M) NEKTMQIEQIVKKVKECSLTPEEGLELI

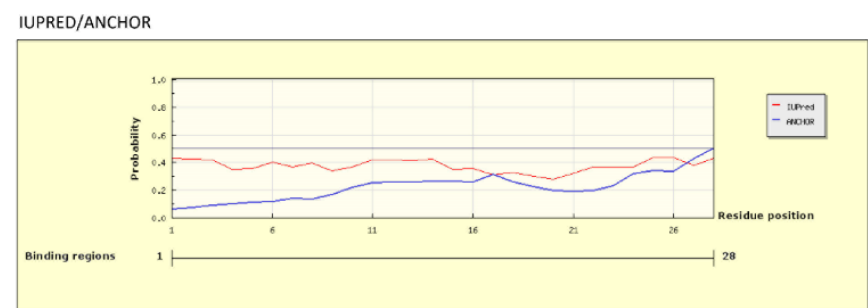

COILED-COIL PREDICTOR

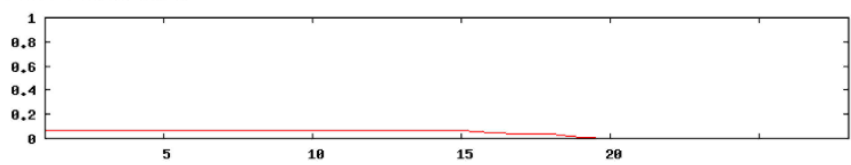

Sequence is too short for PONDR

\section{OBSERVATIONS}

The predicted $\alpha$-helices are not predicted to be interaction regions (ANCHOR). The docking region is predicted to be borderlilne ordered (IUPred/PONDR), and not to form a coiled-coil.

Poor agreement with VirA ${ }^{\mathrm{C} D D}$ 


\section{OzmN ${ }^{\mathrm{C} D D}$}

IUPRED/ANCHOR

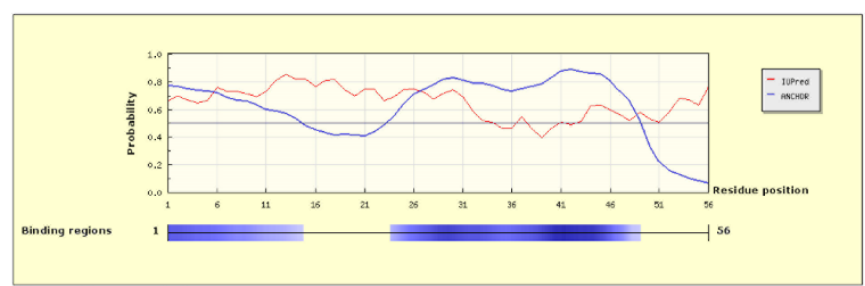

COILED-COIL PREDICTOR
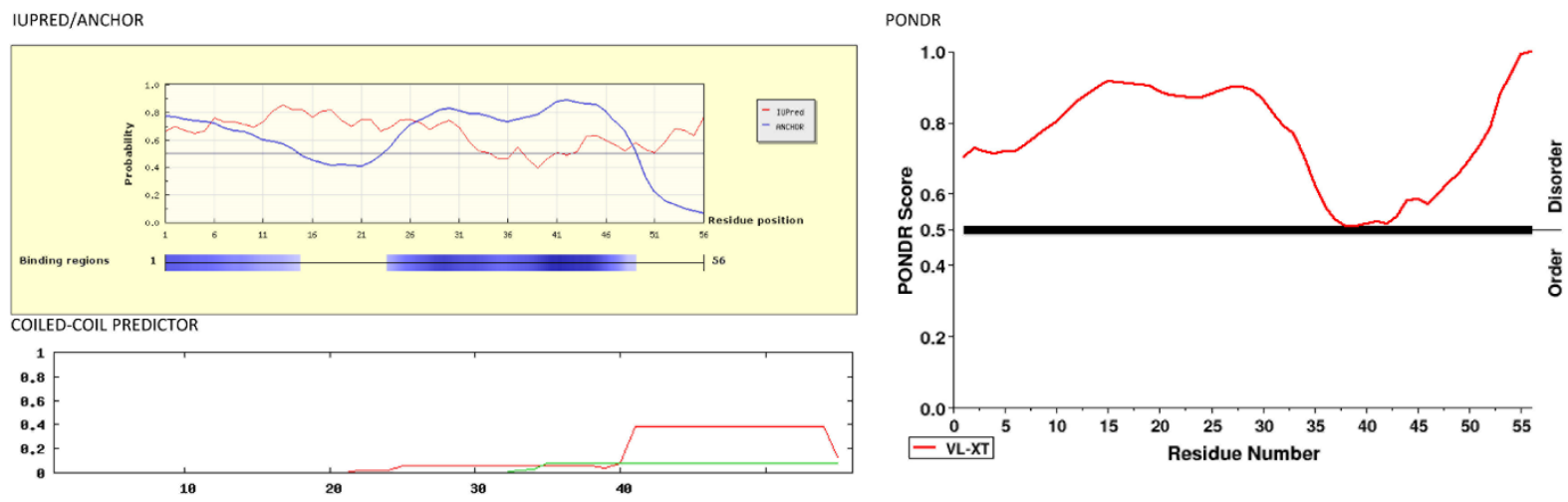

\section{OBSERVATIONS}

Predicted interaction region (ANCHOR) overlaps well with the predicted $\alpha$-helix. The docking region is predicted to be borderline to disordered (IUPred/PONDR), and not to form a coiled-coil.

\section{Agreement with VirA ${ }^{\mathrm{C} D D}$}

\section{OzmH NDD}

$$
6
$$

MRRRSMDDKRALLLKLLASVRQQAAPATAPRRTA

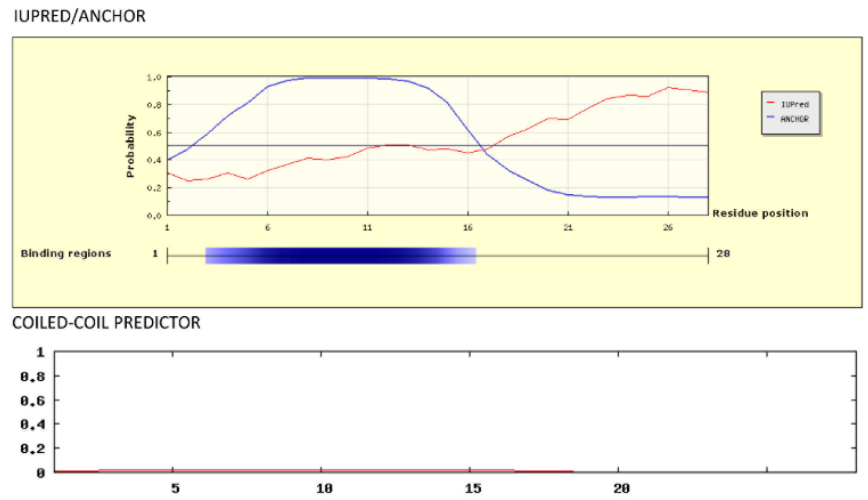

Too short for PONDR

\section{OBSERVATIONS}

Predicted interaction regions (ANCHOR) overlap well with predicted $\alpha$-helix. The docking region is predicted to be borderline disordered (IUPred), but not to form a coiled-coil.

\section{Strong agreement with VirFG ${ }^{\mathrm{N}} D D$}




\section{OzmH ${ }^{\mathrm{C} D D}$}

28

4346

60

IELVGPDEPTAGSGEEPPADGTGDTAAPDELIAKLEARFAAGELSAAEVLDLLDAELATRERR

IUPRED/ANCHOR

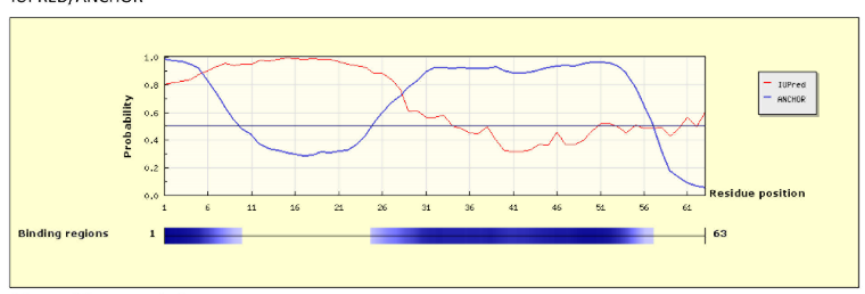

COILED-COIL PREDICTOR

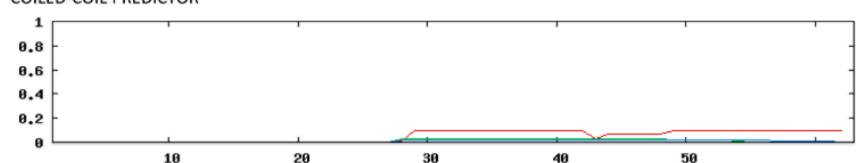

PONDR

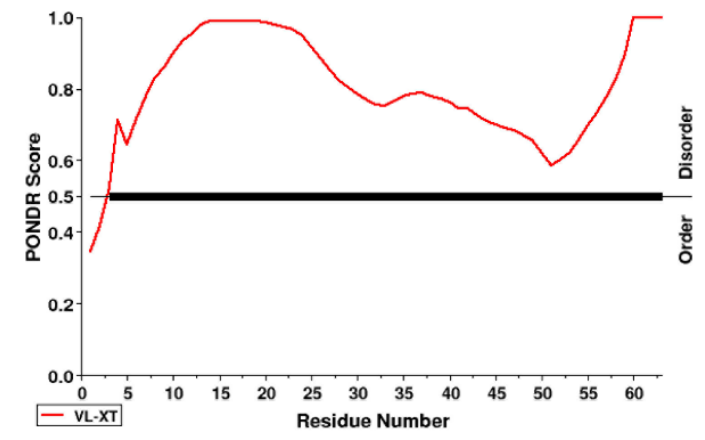

\section{OBSERVATIONS}

Predicted interaction region (ANCHOR) overlaps well with the predicted $\alpha$-helices. The docking region is predicted to be borderline to disordered (IUPred/PONDR), and not to form a coiled-coil. Agreement with VirA ${ }^{\mathrm{C} D D}$

\section{OzmJ NDD}

$3 \quad 16 \quad 20 \quad 29$

MSTAEFRDILDRYARGAVGAEQARDLLAARRPAQAPGRPTAPAPSGQDAATGPADRPETL

IUPRED/ANCHOR

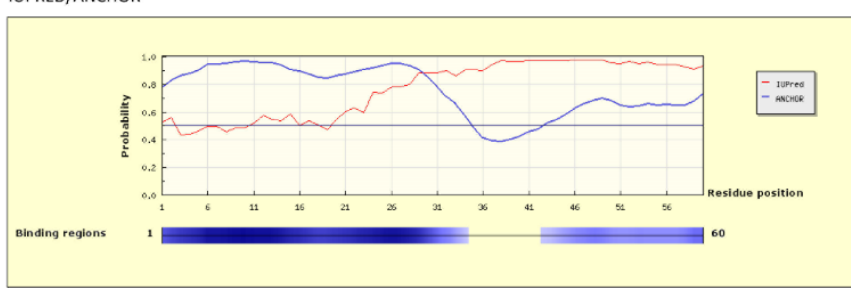

COILED-COIL PREDICTOR

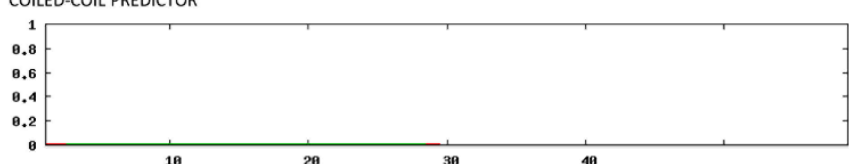

PONDR

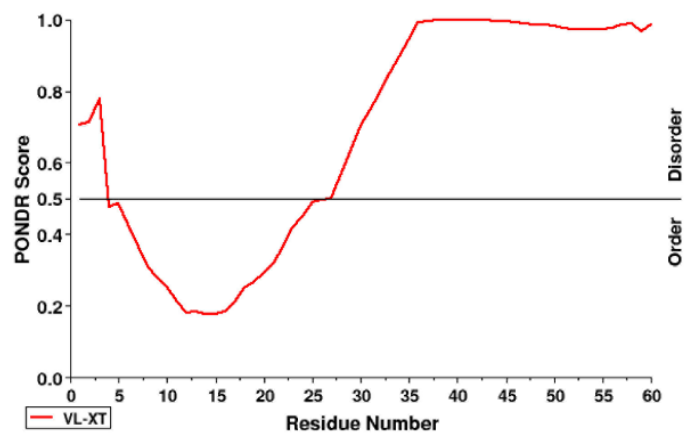

OBSERVATIONS

Predicted interaction regions (ANCHOR) overlap well with predicted $\alpha$-helices. The docking region is predicted to be borderline disordered (IUPred) to somewhat ordered (PONR) but not to form a coiled-coil.

Agreement with VirFG ${ }^{\mathrm{N}} \mathrm{DD}$ 


\section{OzmK ${ }^{C} D D$}

$2 \quad 8 \quad 12 \quad 19$

EAELVERIEELPEDTVATLLA

IUPRED/ANCHOR

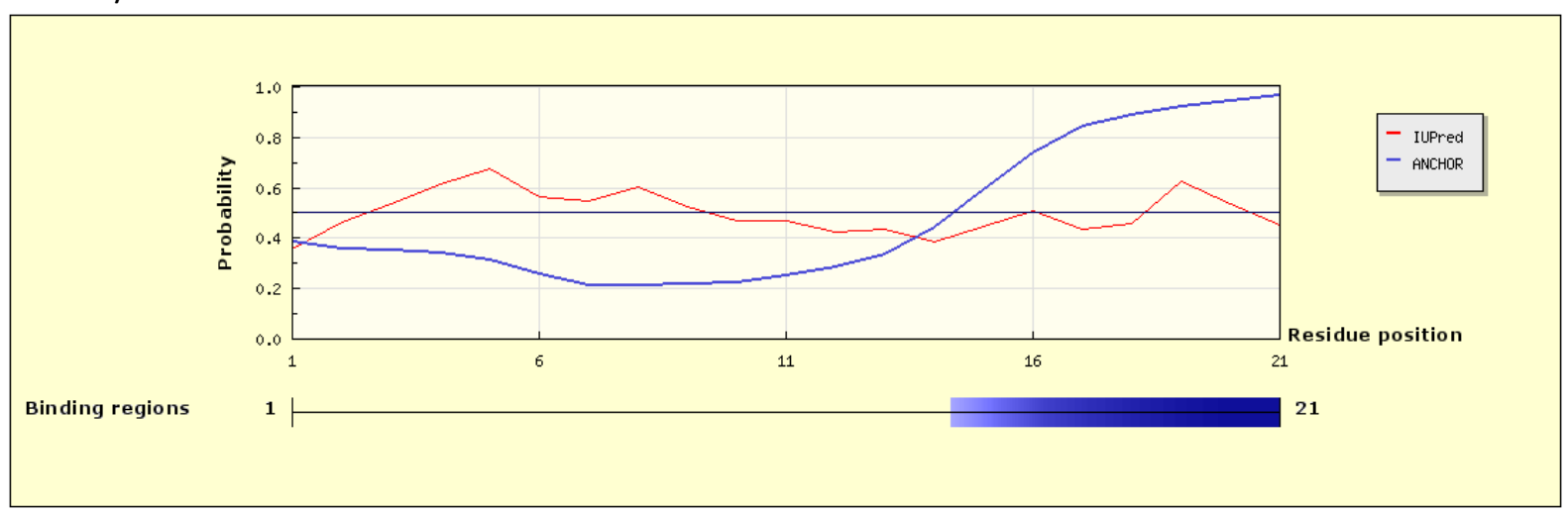

Sequence is too short for PONDR/COILED-COIL PREDICTOR

\section{OBSERVATIONS}

The second $\alpha$-helix predicts to be an interaction region (ANCHOR). The docking region is predicted to be borderline disordered (IUPred).

Some agreement with VirA ${ }^{C} D D$

\section{OzmL NDD}

$3 \quad 13 \quad 16 \quad 29$

MSREDLATSRKARLSPQKRALLEKLSGAARTSGTTRIPR

IUPRED/ANCHOR

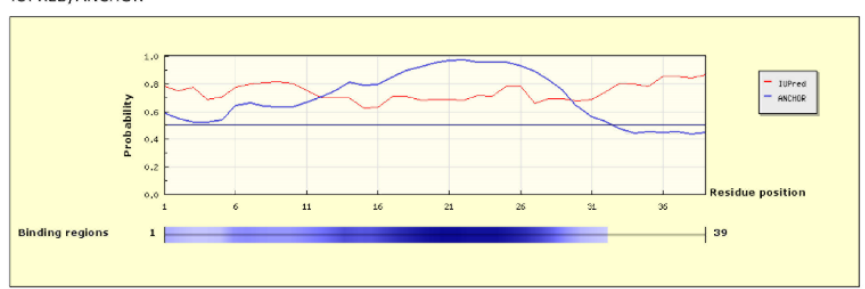

COILED-COIL PREDICTOR

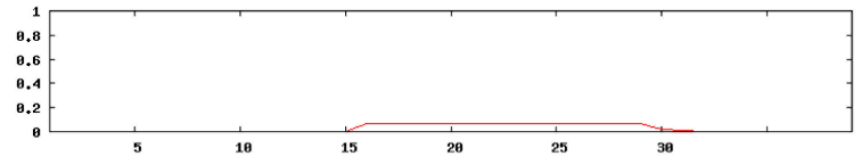

OBSERVATIONS

Predicted interaction regions (ANCHOR) overlap well with predicted $\alpha$-helices. The docking region is predicted to be disordered (IUPred/PONDR), but not to form a coiled-coil.

Strong agreement with VirFG ${ }^{N} D D$

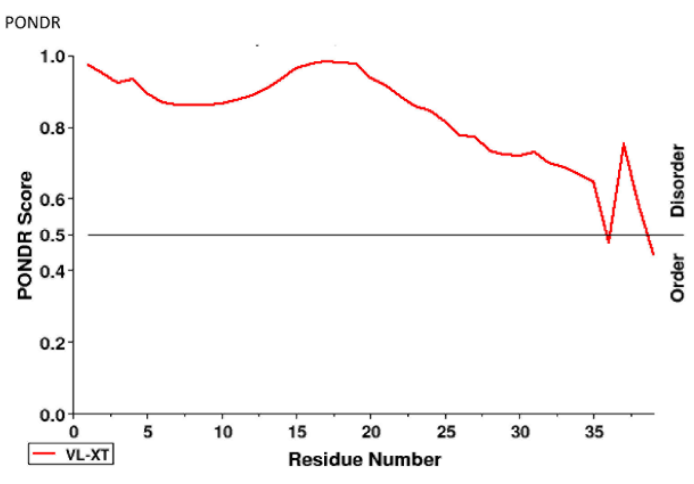




\section{RhiC ${ }^{\mathrm{D} D D}$}

39

48

53

60

ADNQRTAMAVSPNDLDHAVSAGNNVRGQSRPIEIHPPFADQLEGLFLSGELSIDSLLNIVSPGVAEIR

EVSI

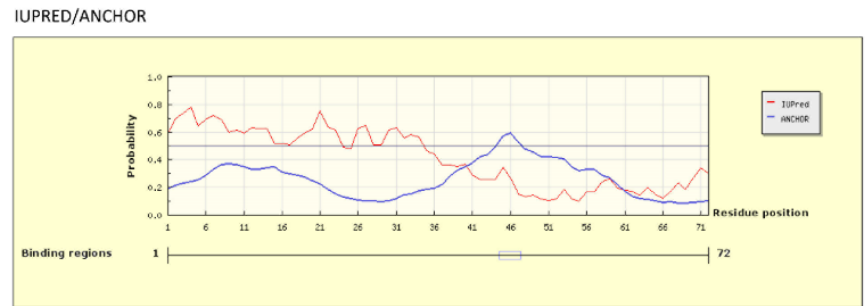

COILED-COIL PREDICTOR
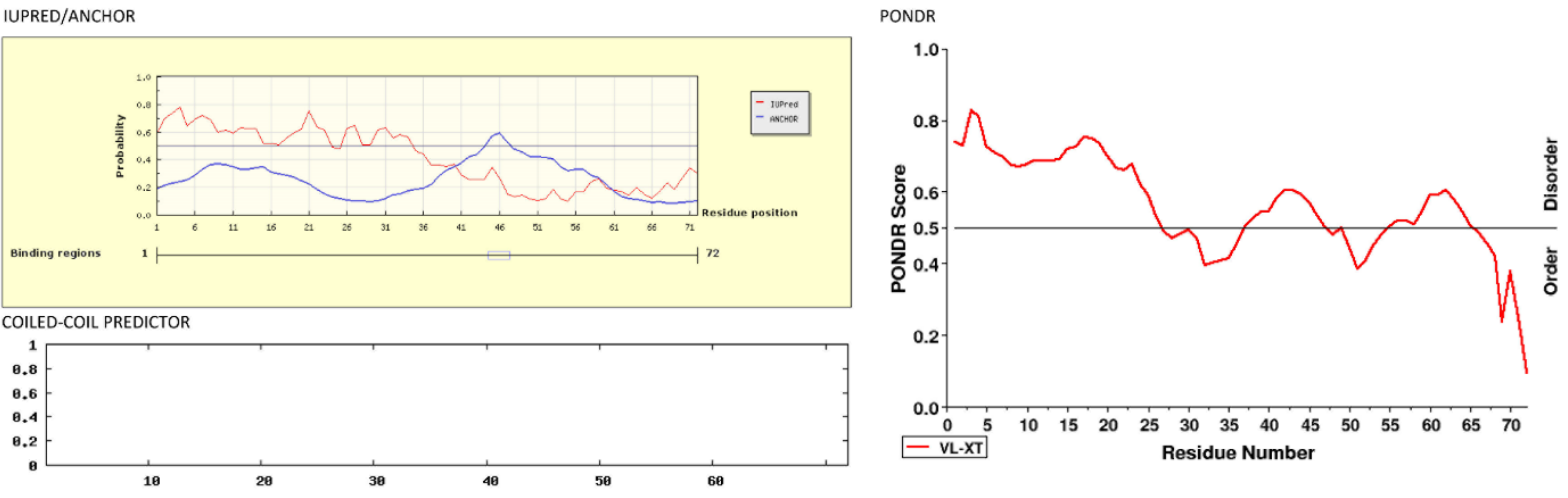

\section{OBSERVATIONS}

The secondary structure elements are not predicted to be interaction regions (ANCHOR). The docking region is predicted to be borderline disordered (PONDR) to ordered (IUPred), and not to form a coiled-coil.

Poor agreement with VirA ${ }^{C} D D$

\section{RhiD NDD}
2
12
19
36

(M) SSQEFQSIIAGLQNRQITVAEAKQRLQRLKAASATLGTEQTEAADKAVSGTGRAQEP
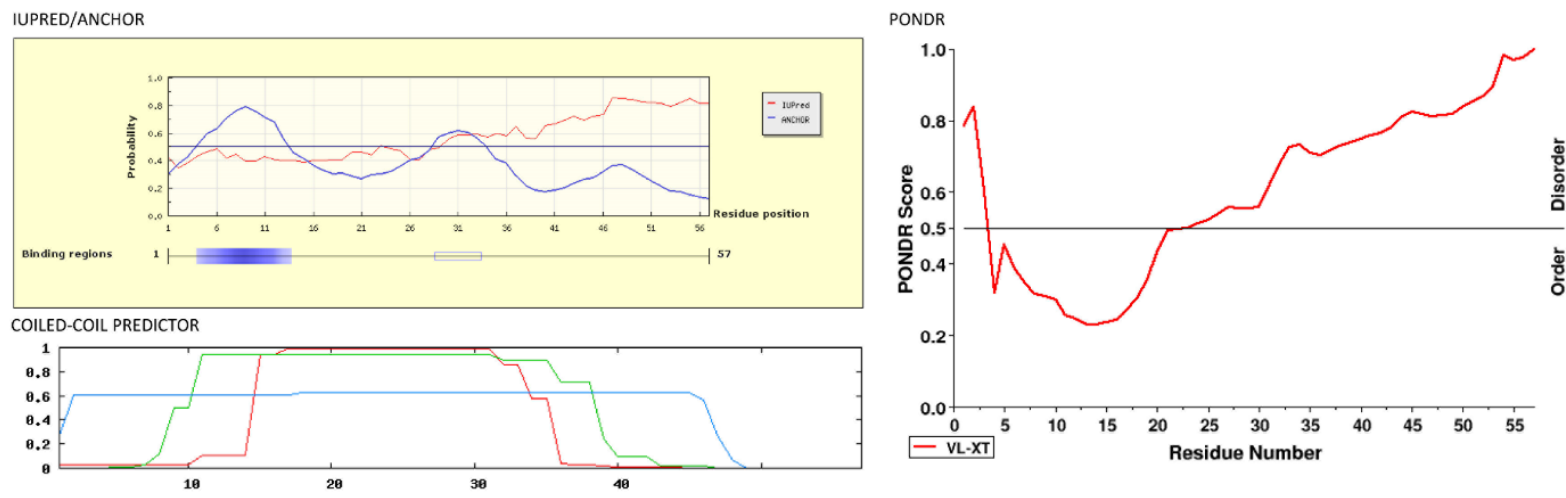

\section{OBSERVATIONS}

The first predicted $\alpha$-helix is predicted to be an interaction region, but the second only weekly. The docking region is predicted to be mixed ordered/disordered (IUPred/PONDR) and strongly predicted to form a coiled-coil.

Does not agree with VirFG NDD; may be an alternative type of docking domain? 


\section{$\operatorname{RizC}{ }^{C} D D$}

29

37

40

RALVTEAPPRGATQSANGHALEGMSDGNLEELFARLSGVSSQKGEA

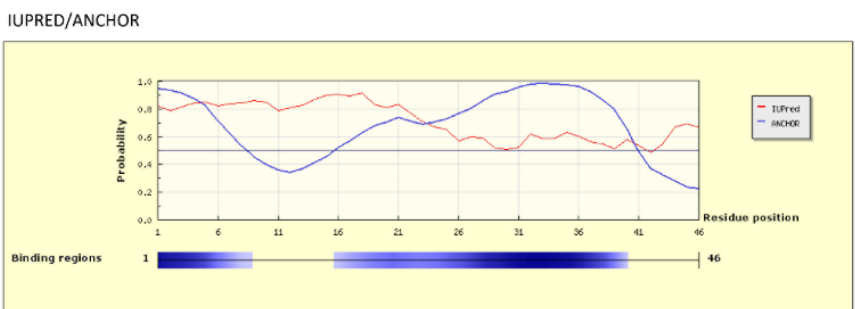

COILED-COIL PREDICTOR
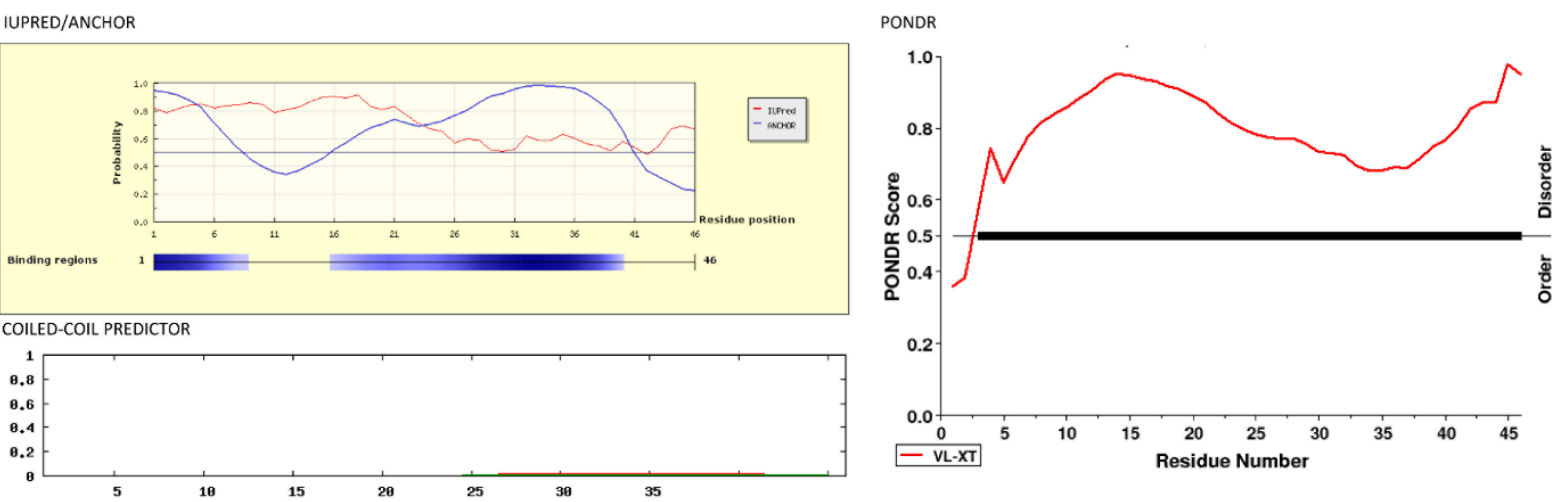

\section{OBSERVATIONS}

The predicted $\alpha$-helices are predicted to be an interaction region (ANCHOR). The docking region is predicted to be borderline (IUPred) to strongly ordered (PONDR), and not to form a coiled-coil. Agreement with VirA ${ }^{\mathrm{C} D D}$

\section{RizD NDD}
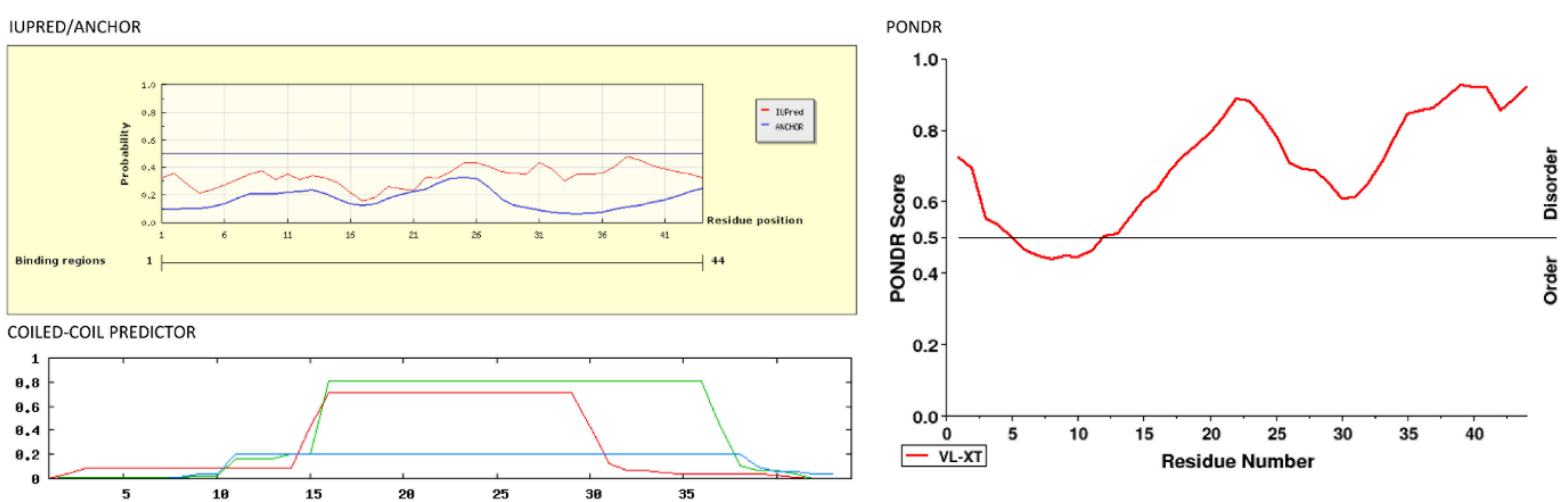

\section{OBSERVATIONS}

The predicted $\alpha$-helix is not predicted to be an interaction region (ANCHOR). The docking region is predicted to be borderline disordered (IUPred) to disordered (PONDR), but to form a coiled-coil. Does not agree with VirFG NDD; may be an alternative type of docking domain? 
Ta-1 ${ }^{\mathrm{C} D D}$

21

29

35

44

GNEIQKTVSAGAPAQGEPPSLDELLRQVEAGELDPSVAQQFLTNSQS

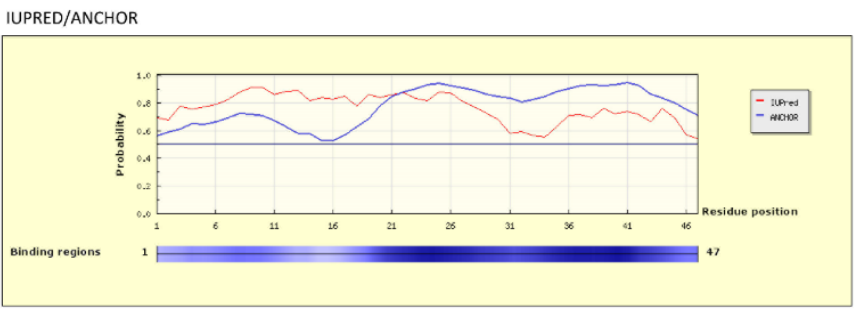

COILED-COIL PREDICTOR

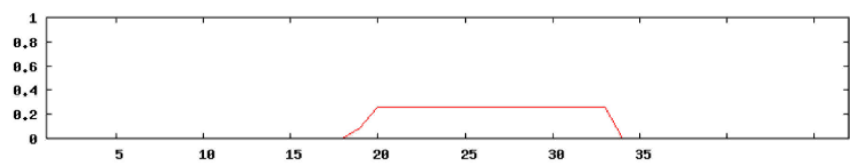

PONDR

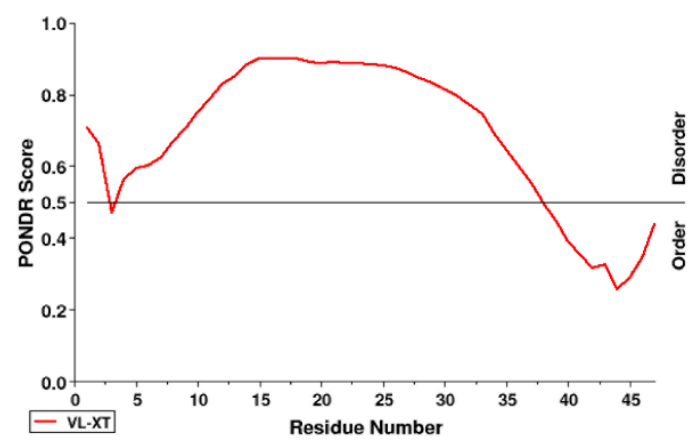

\section{OBSERVATIONS}

The predicted $\alpha$-helices are predicted to be interaction regions (ANCHOR). The docking region is predicted to be disordered (IUPred) to mixed disordered/ordered, and not to form a coiled-coil. Agreement with VirA ${ }^{\mathrm{C} D D}$

\section{TaO NDD}

(M) GEGHRIGVLQQP PRGRDCHEADDGSRRI PQP PVPQVGRPGRGAVMTRLEILQALQSGRMALADAK

IUPRED/ANCHOR

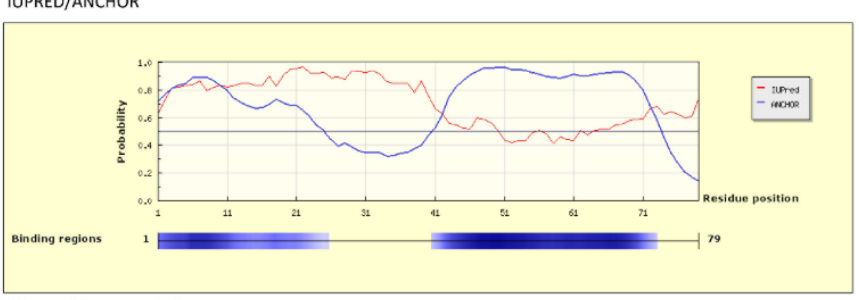

COILED-COIL PREDICTOR

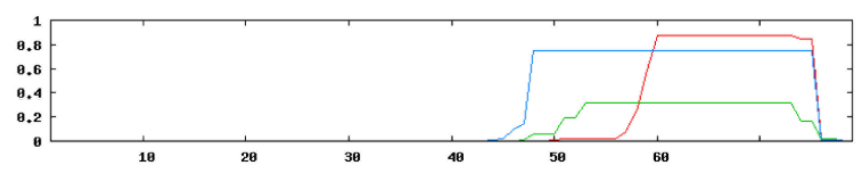

PONDR

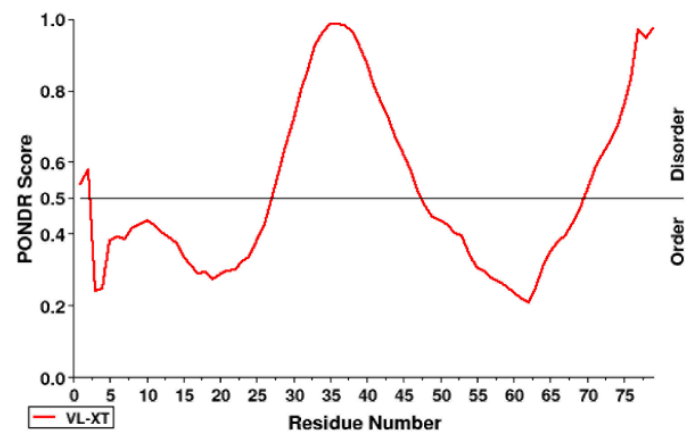

\section{OBSERVATIONS}

The predicted $\alpha$-helices are predicted to be interaction regions (ANCHOR). The docking region is predicted to be borderline (IUPred) to mixed ordered/disordered (PONDR), and the second $\alpha$-helix to form a coiled-coil.

Does not agree with VirFG NDD; may be an alternative type of docking domain? 


\section{TaiD CDD}

64

AERIARDGGRRAAEAARAPDAAMASDVAEASVVSEATEASDASEASDASEASEASEASEASKAPADLA ALLERFRAGQMDLDDIVDLV

$\begin{array}{lll}74 & 81 \quad 87\end{array}$

IUPRED/ANCHOR

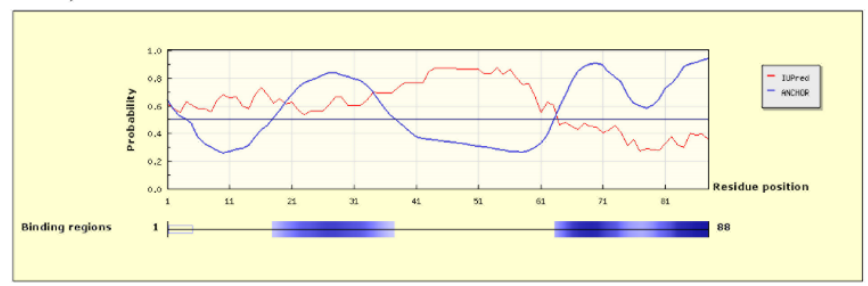

COILED-COIL PREDICTOR
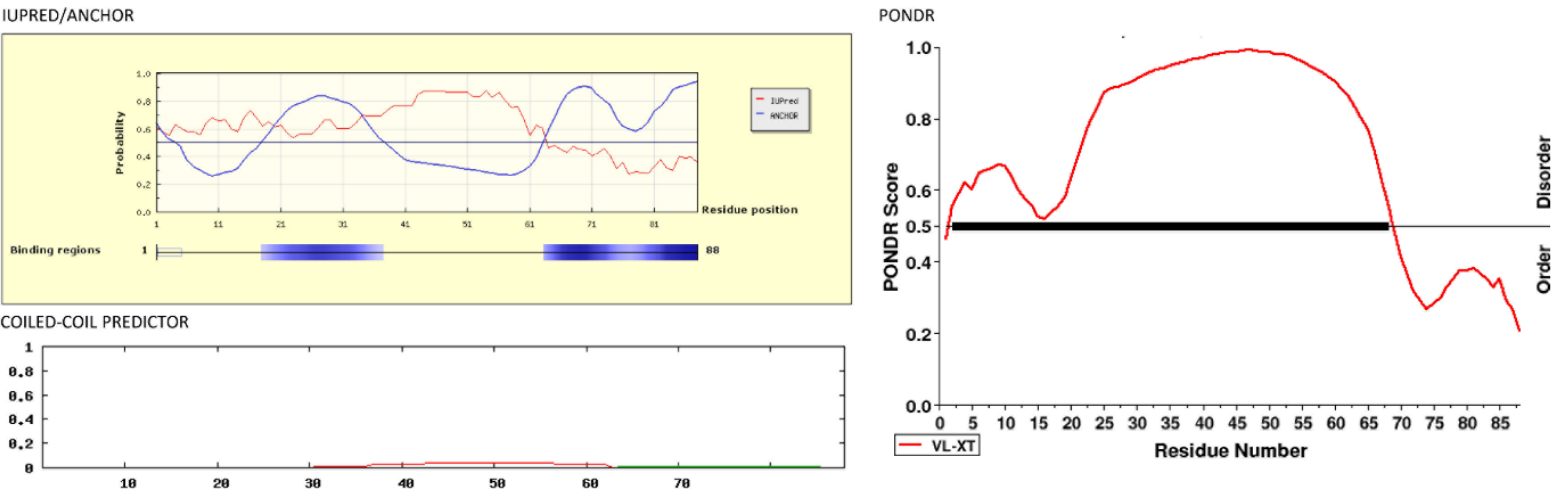

OBSERVATIONS

Predicted interaction region (ANCHOR) overlaps well with the predicted $\alpha$-helices. The docking region is not predicted to be disordered (IUPred/PONDR), nor to form a coiled-coil.

\section{Strong agreement with VirA ${ }^{\mathrm{C}} \mathrm{DD}$}

\section{TaiE NDD}

MPRWRPTWLKRLSCPKQPRHPTHPKHPTHPKHPKHPKHPRHPKHPRISRRCSSASARGKWISTTSSTW FDGDHVNKKDILLAYREGLLDTGSAQRVLDALRERSASAP
76

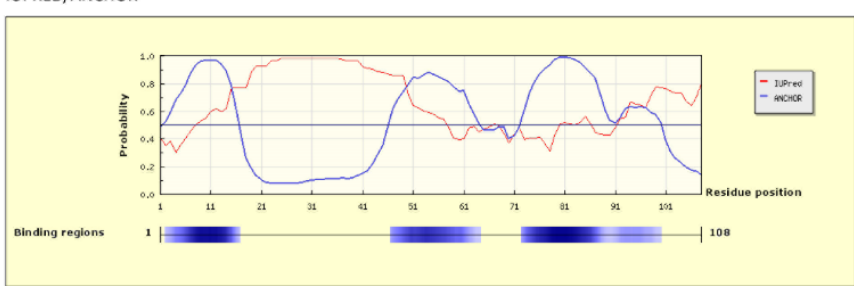

COILED-COIL PREDICTOR
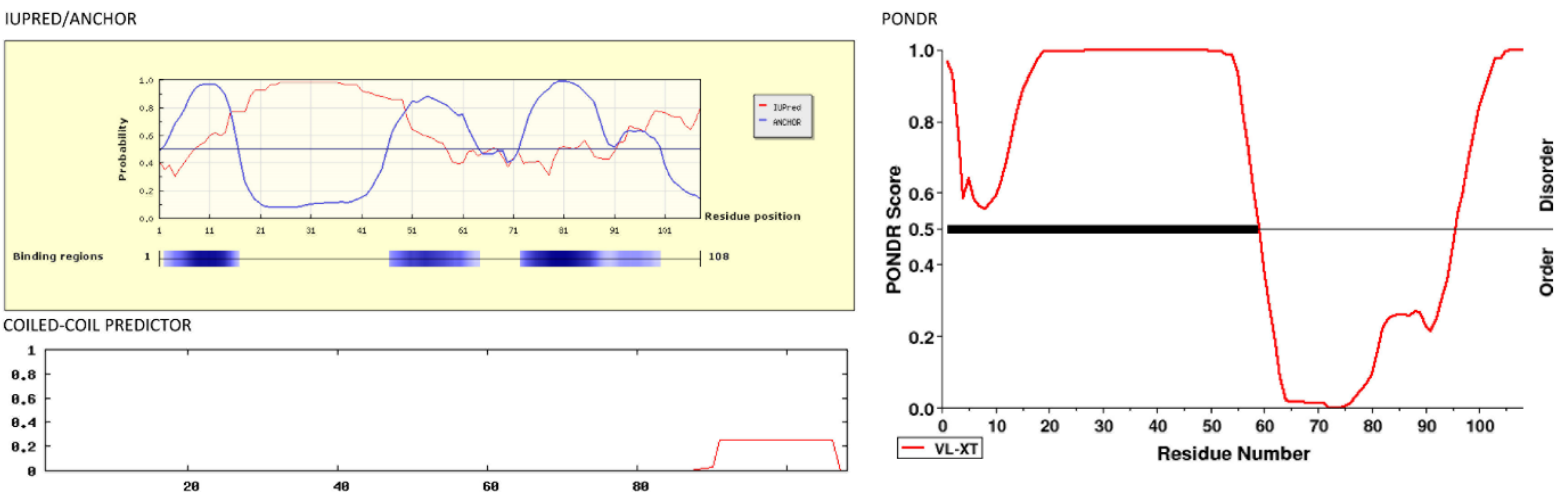

OBSERVATIONS

Predicted interaction regions (ANCHOR) overlap well with predicted $\alpha$-helices. The docking region is predicted to be borderline disordered (IUPred) or ordered (PONDR) and not to form a coiled-coil. Agreement with VirFG ${ }^{\mathrm{N}} \mathrm{DD}$ 


\section{TaiE CDD}

53

62

GTQLGARLPAAQAARAGAFAGVEPGEPDARALPAAARAAAPPAHTDAAAHTDTDALLRAIERGELDAG DADAIWRRMQSRAARPEPLAQP

70

IUPRED/ANCHOR

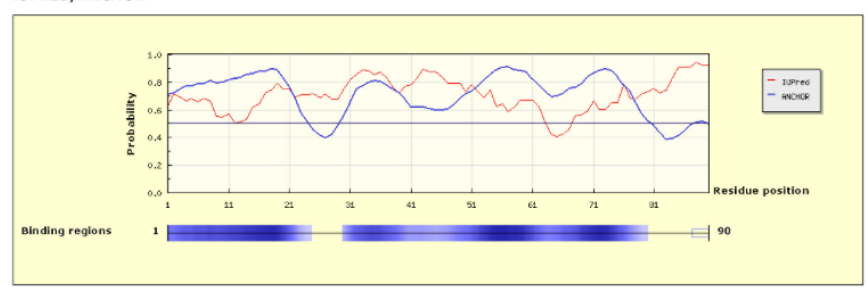

COILED-COIL PREDICTOR
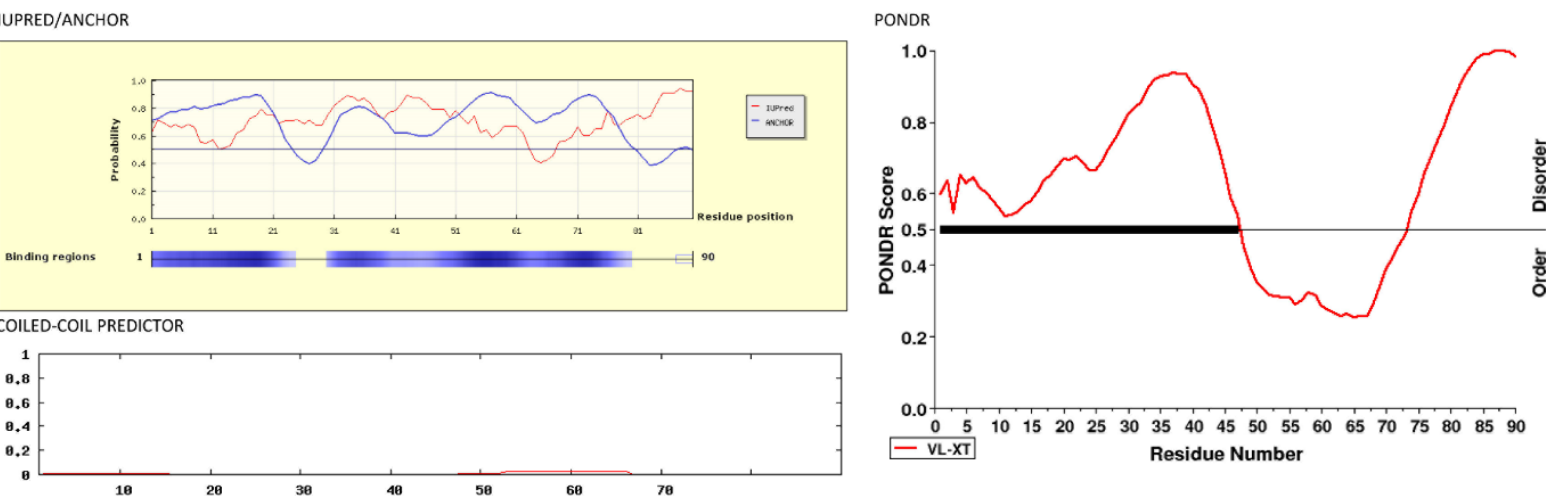

OBSERVATIONS

Predicted interaction region (ANCHOR) overlaps well with the predicted $\alpha$-helices. The docking region is not predicted to be somewhat disordered (IUPred) to largely ordered (PONDR), nor to form a coiled-coil.

Agreement with VirA ${ }^{\mathrm{D} D D}$

\section{TaiK NDD}
3
11
17
35

(M) RSSKEIFEALRDGRLSREEAHAALRSARAAADAALGASGAFEPRGAEAAGANGAADAGDAASGAS AANGERAAHAAKTAESAAAADA
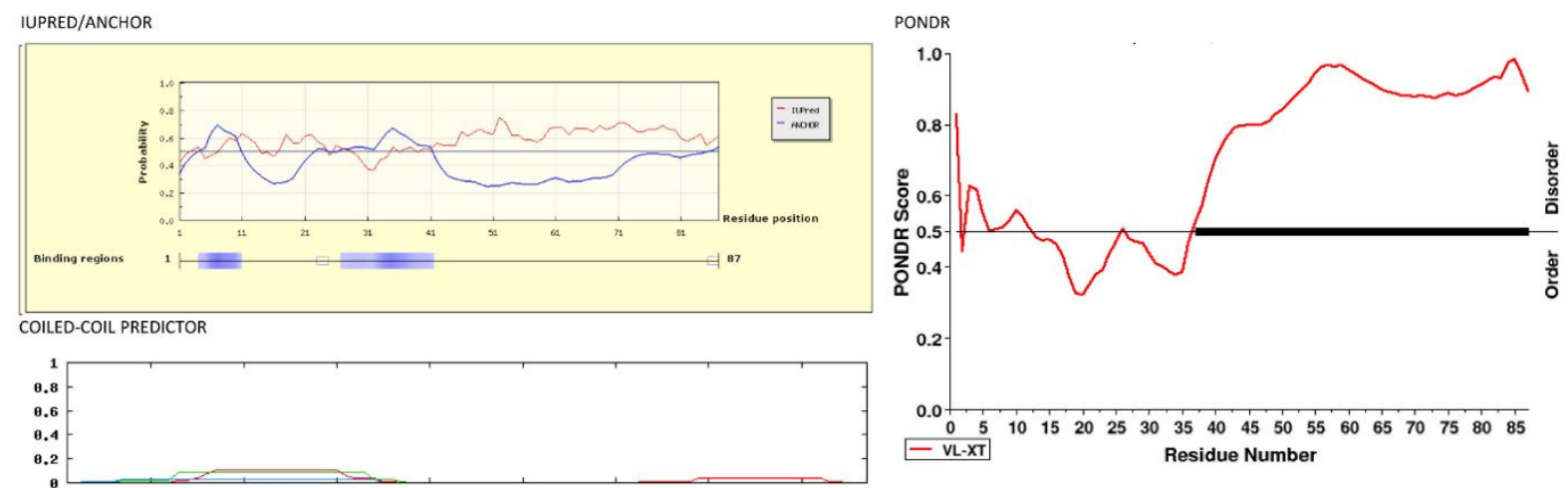

\section{OBSERVATIONS}

Predicted interaction regions (ANCHOR) overlap well with predicted $\alpha$-helices (though predicted binding regions are week). The docking region is predicted to be borderline disordered (IUPred/PONDR) and not to form a coiled-coil.

Agreement with VirFG ${ }^{\mathrm{N}} \mathrm{DD}$ 


\section{Tail ${ }^{C} D D$}

ASELALARRRAGGAPLPDAEAASATTATTASASGTAHAPNAAGDAHAPNAAGARAATPAPAENAAGAV HAARAANPANPATLANPAPGGGVPLDDVLARVHRGELSVEAAEALLAGALG

$93 \quad 102105$

IUPRED/ANCHOR

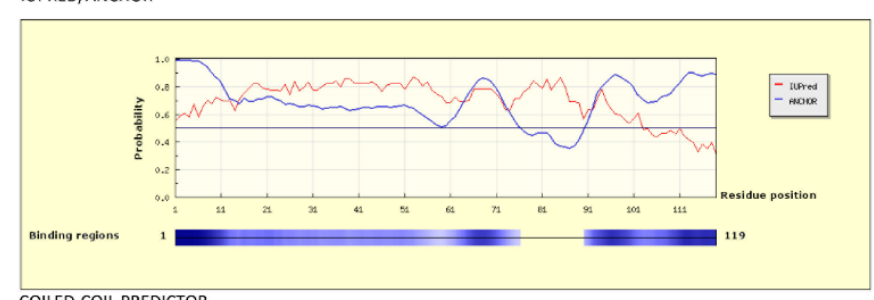

COILED-COIL PREDICTOR

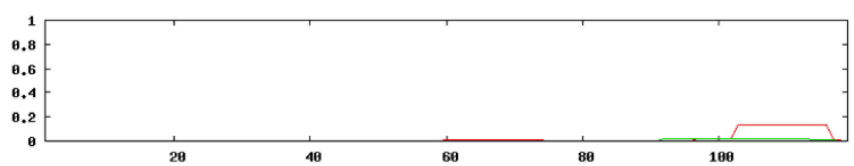

OBSERVATIONS

Predicted interaction region (ANCHOR) overlaps well with the predicted $\alpha$-helices. The docking region is predicted to be mixed ordered/disordered (IUPred/PONDR), and not to form a coiled-coil. Agreement with VirA ${ }^{\mathrm{C} D D}$

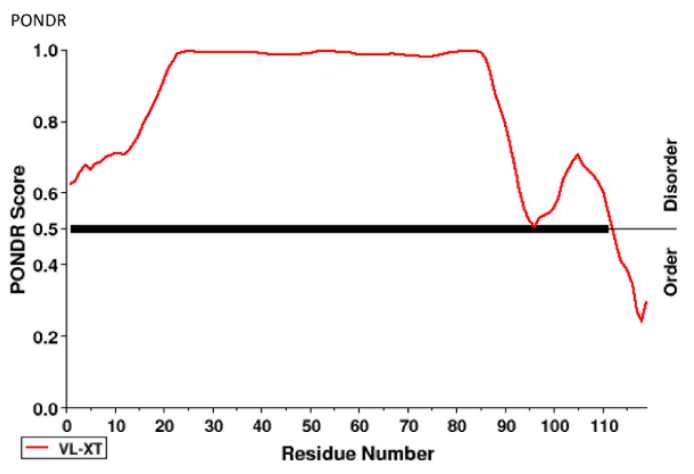

\section{TaiM NDD}

3

IUPRED/ANCHOR

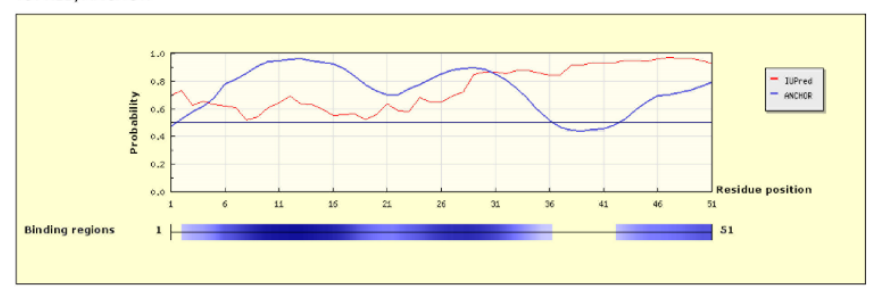

COILED-COIL PREDICTOR

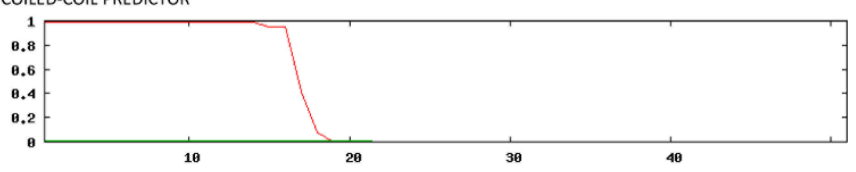

OBSERVATIONS

Predicted interaction regions (ANCHOR) overlap well with predicted $\alpha$-helices (though predicted binding regions are week). The docking region is predicted to be borderline (IUPred) to strongly disordered (PONDR), and not strongly predicted to form a coiled-coil.

Agreement with VirFG ${ }^{\mathrm{N}} \mathrm{DD}$ 


\section{$5923^{C} D D$}

26

32

$45 \quad 48 \quad 51$

ARGALAELFAPA IVAAPAQLANAAADASLGDHSAAELARI LAHELGGLESRGAL

IUPRED/ANCHOR

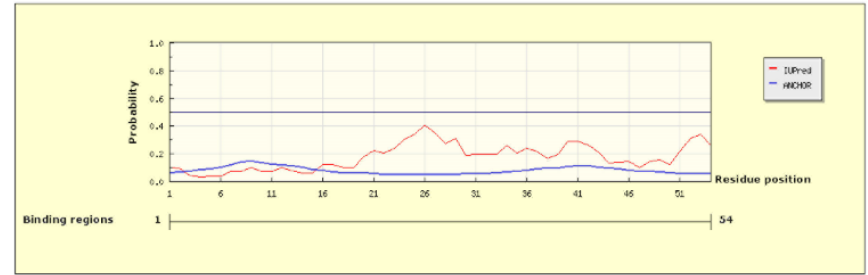

COILED-COIL PREDICTOR

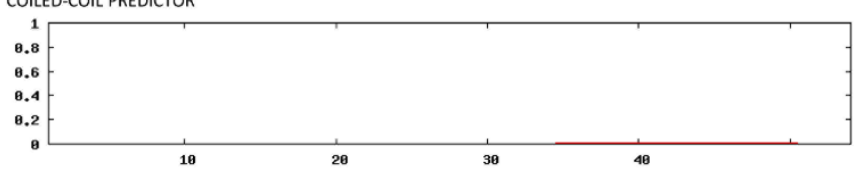

OBSERVATIONS

The predicted $\alpha$-helices are not predicted to be interaction regions (ANCHOR). The docking region is predicted to be ordered (IUPred) to borderline disordered (PONDR), and not to form a coiled-coil. Poor agreement with VirA ${ }^{\mathrm{C}} \mathrm{DD}$

\section{NDD}

4

\section{MTDMDKDLLLQSIQTIRELKTRLAQAEQGHH}

IUPRED/ANCHOR

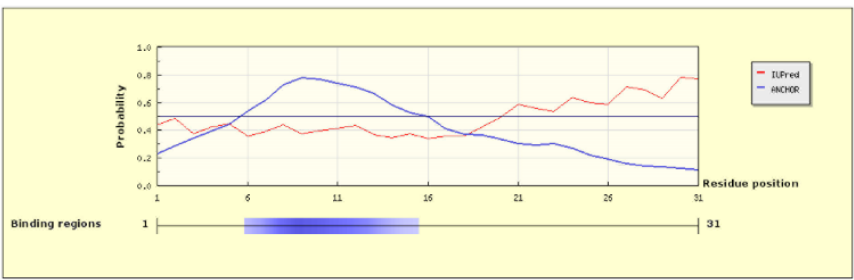

COILED-COIL PREDICTOR
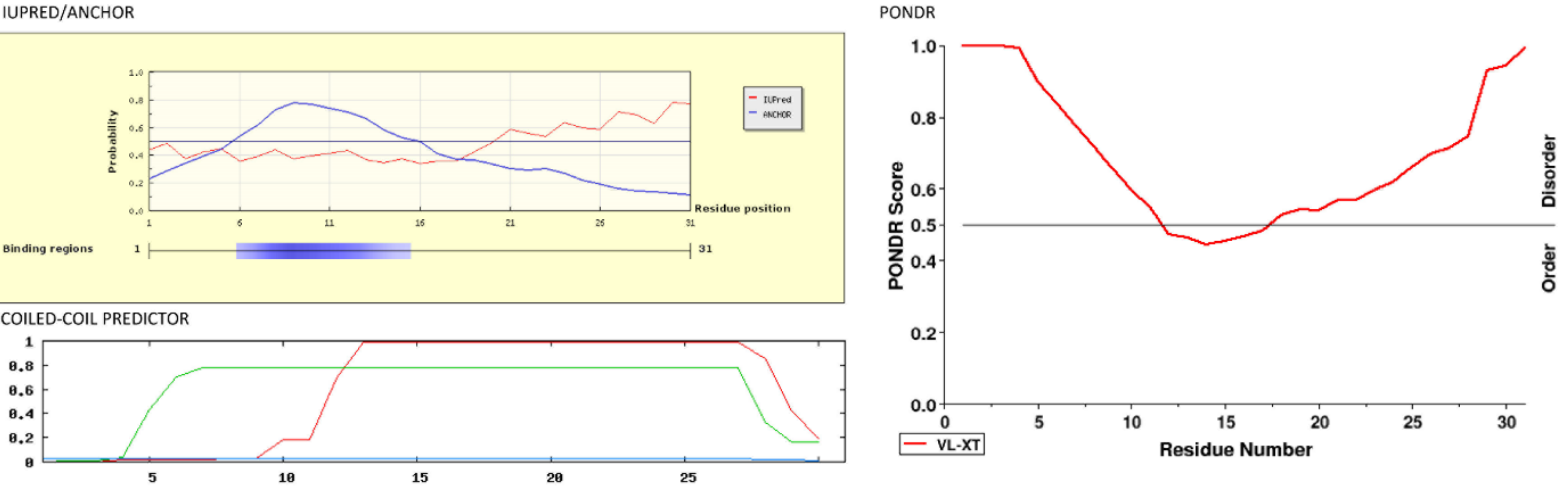

\section{OBSERVATIONS}

The predicted $\alpha$-helix is predicted to be an interaction region. The docking region is predicted to be mixed ordered/disordered (IUPred/PONDR) but also predicted to form a coiled-coil.

Does not agree with VirFG ${ }^{\mathrm{N}} \mathrm{DD}$; may be an alternative type of docking domain? 


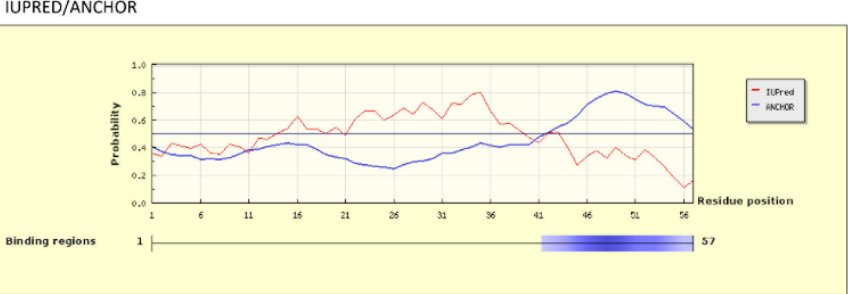

COILED-COIL PREDICTOR
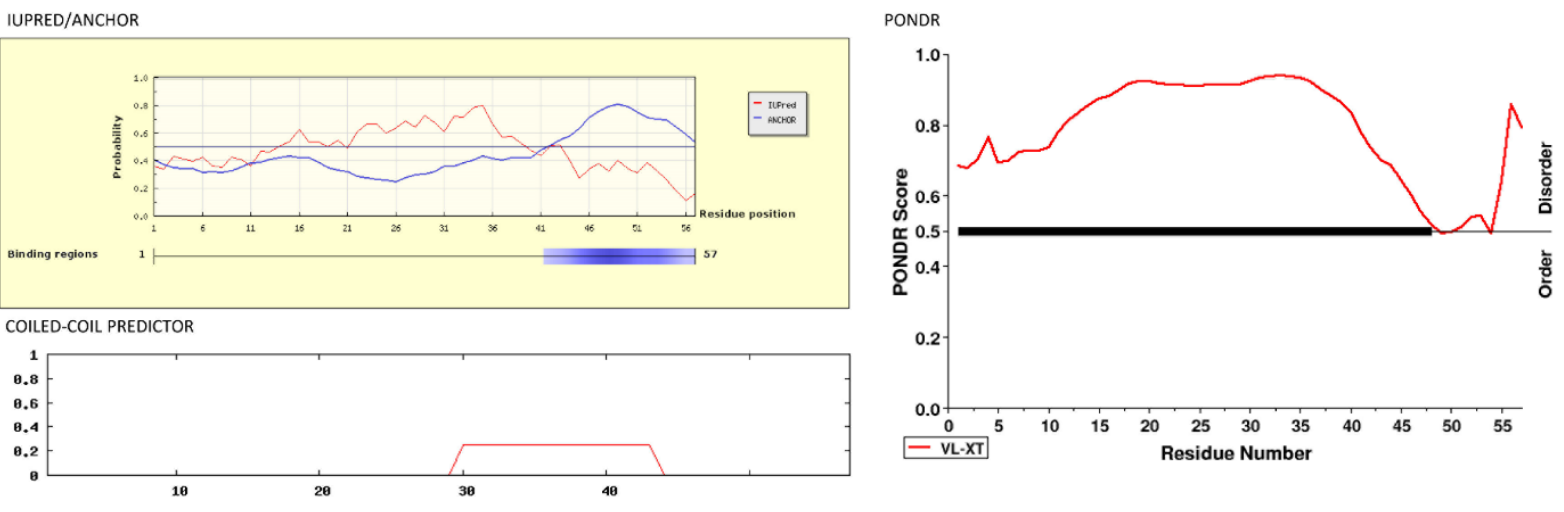

\section{OBSERVATIONS}

The second predicted $\alpha$-helix is predicted to be an interaction region (ANCHOR). The docking region is predicted to be mixed ordered/disordered (IUPred/PONDR), and not to form a coiled-coil. Some agreement with VirA ${ }^{C} D D$

\section{NDD}

11

40

\section{MNKPTSSDGWKDDYLSRLSRLSKNQLMALALKLKQQQLEQGPAA}
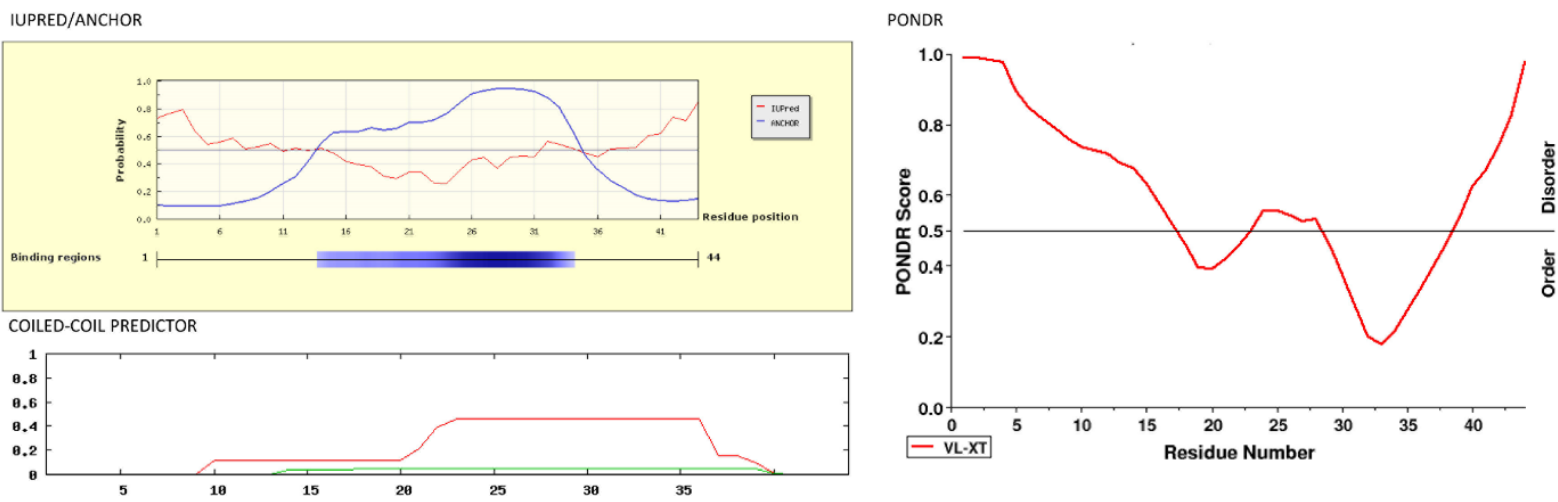

\section{OBSERVATIONS}

Predicted interaction regions (ANCHOR) overlap well with the predicted $\alpha$-helix. The docking region is predicted to be borderline disordered to more ordered (IUPred/PONDR), and not strongly predicted to form a coiled-coil.

Agreement with VirFG ${ }^{\mathrm{N} D D}$ 
Key:

Red $=\alpha$-helices observed in the NMR structure of the DEBS $2{ }^{\mathrm{C} D D} / \mathrm{DEBS} 3{ }^{\mathrm{N}} \mathrm{DD}$ complex

Blue $=\alpha$-helices predicted by PsiPred

\section{DEBS $2{ }^{\mathrm{C} D D}$}
8
20
28
42

70

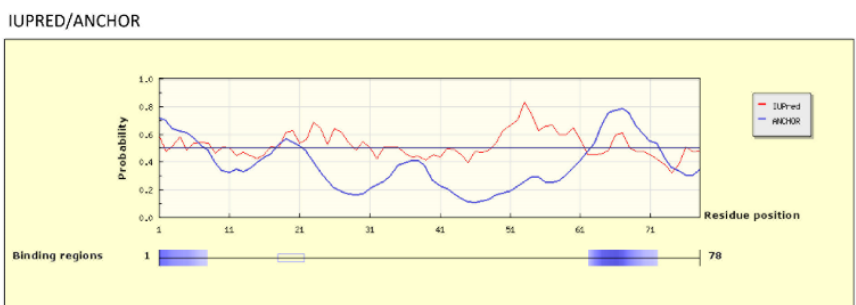

PONDR
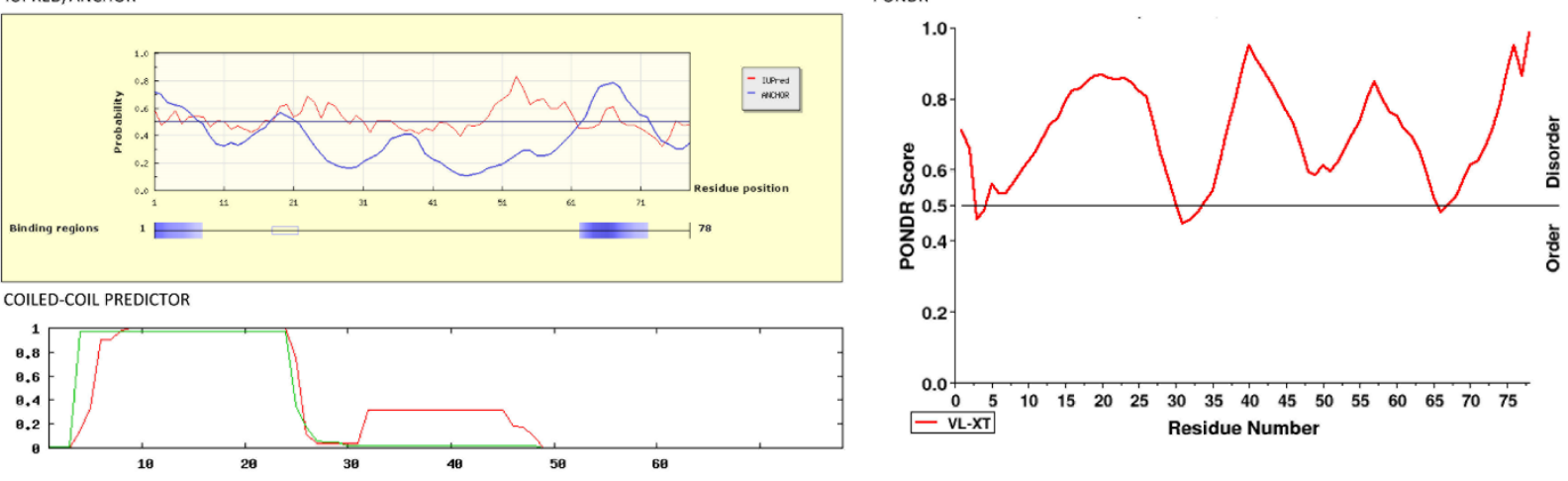

\section{OBSERVATIONS}

The third docking $\alpha$-helix is correctly predicted to be interaction region (ANCHOR), and to be relatively disordered (IUPred/PONDR). The first two $\alpha$-helices, which form a dimerization motif (four $\alpha$-helical bundle), are predicted to be borderline disordered (IUPred) to disordered (PONDR), and to form a coiled-coil motif.

\section{DEBS 3 NDD}

\section{0

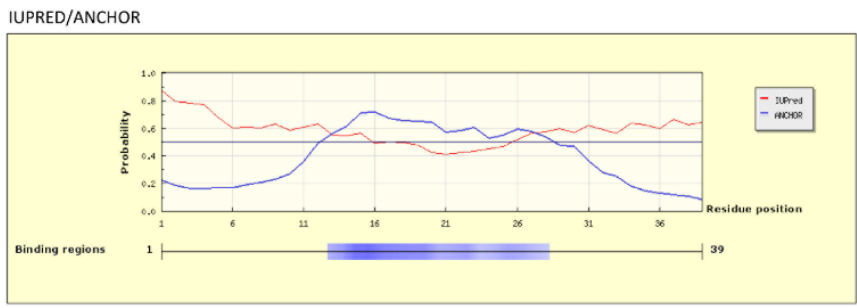

COILED-COIL PREDICTOR

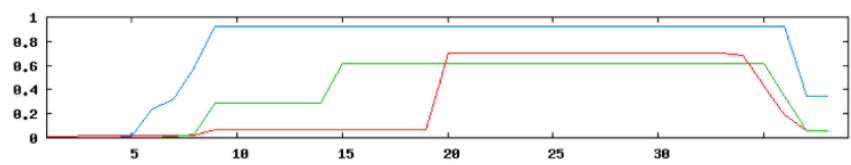

PONDR

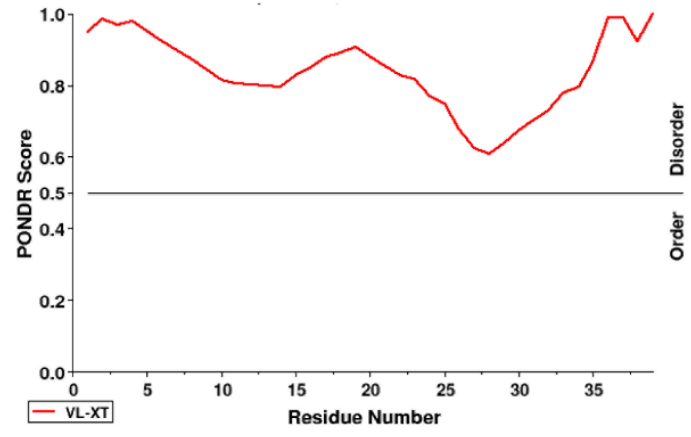


OBSERVATIONS

This region forms a coiled-coil (correctly predicted by the coiled-coil predictor). It is also correctly predicted (ANCHOR) to form an interaction motif, and by both IUPred and PONDR to be disordered (consistent with its coiled-coil nature). 
A

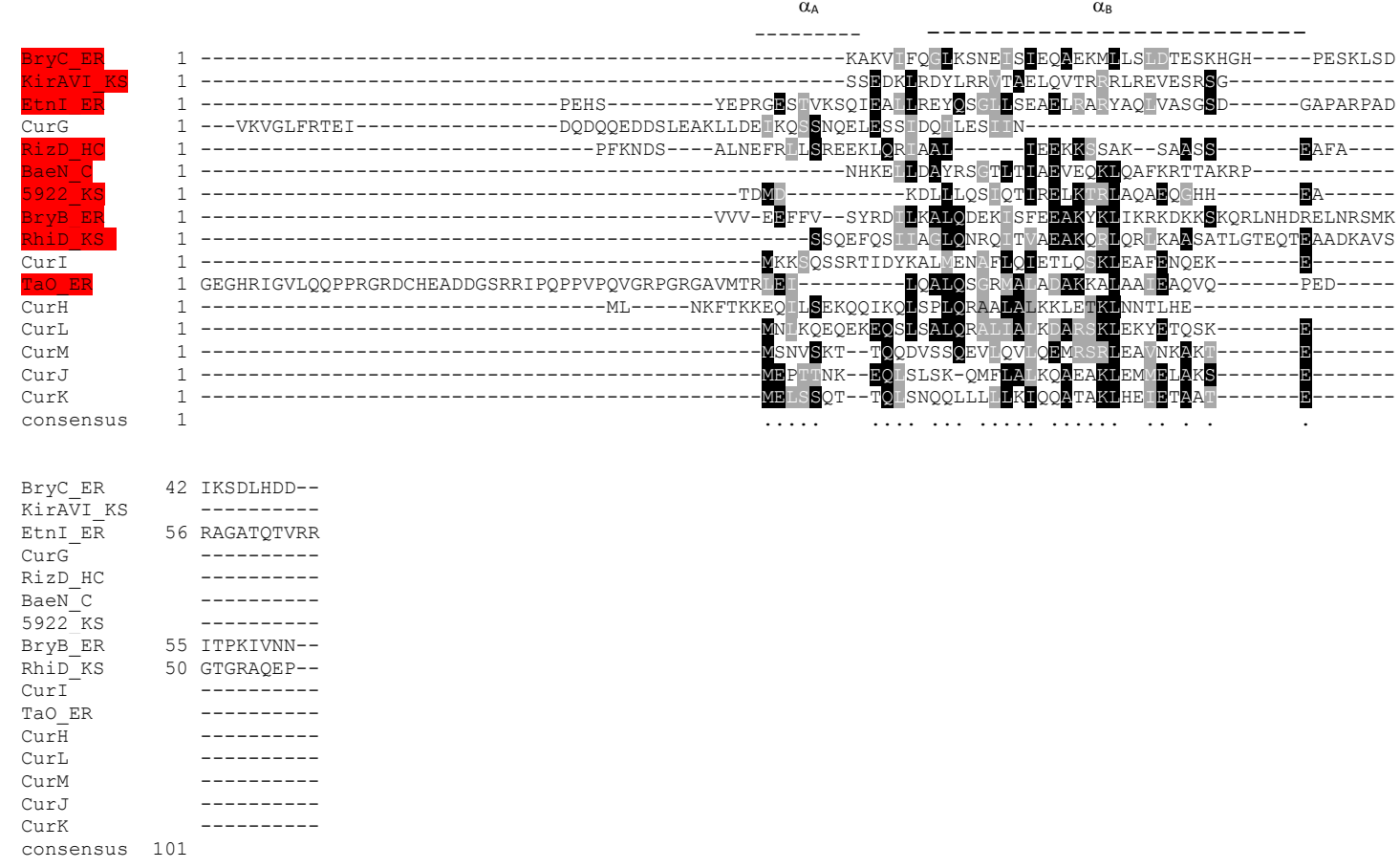

B

CurL

CurJ

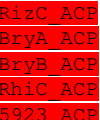

CurI
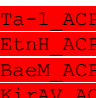

CurG

CurH

Curk

consensus

CurL
CurJ

RizC ACP

BryA ACP

BryB ACP

RhiC ACP

5923 ACP

CurI

Ta-1_ACP

EtnH ACP

Baem ACP

KirAV ACP

KirAV _

Curg

CurH

Curk

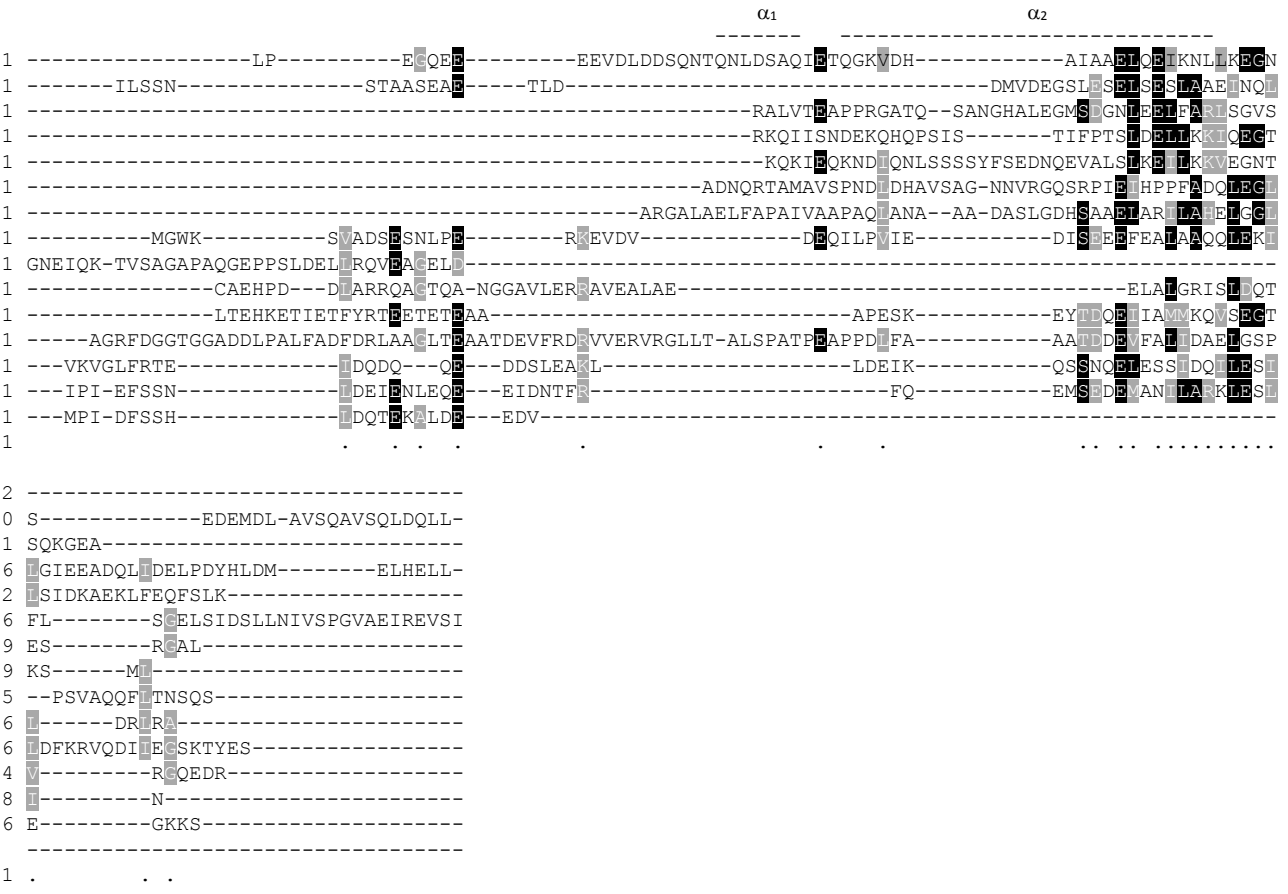

Figure 1. Comparative sequence analysis of docking domains from trans-AT PKS and Class 2 docking domains. (A) Multiple alignment using ClustalOmega ${ }^{11}$ of the $\mathrm{N}$-terminal regions of PKS subunits from trans-AT PKSs (in red), with those of Class $2 \mathrm{~N}$-terminal docking domains of cis-AT PKS. ${ }^{12}$ The positions of the two $\alpha$-helices in the solved structure of the CurH and CurL N-terminal docking domains are indicated, the second of which forms a coiled-coil. (B) Multiple alignment using ClustalOmega ${ }^{11}$ of the C-terminal regions of PKS subunits partenering those in (A) (in red), with those Class 2 C-terminal docking domains of cis-AT. The positions of the two $\alpha$-helices in the solved structure of the CurG and CurK C-terminal docking domains are indicated. Only Ta-1 does not align particularly well with the others. 


\section{ASSOCIATED REFERENCES}

(1) Dosztányi, Z.; Csizmok, V.; Tompa, P.; Simon, I. Bioinformatics 2005, 21, 3433.

(2) Dosztányi, Z.; Mészáros, B.; Simon, I. Bioinformatics 2009, 25, 2745.

(3) Romero, P.; Obradovic, Z.; Li, X.; Garner, E.C.; Brown, C.J.; Dunker, A.K. Proteins 2001, 42, 38.

(4) Li, X.; Romero, P.; Rani, M.; Dunker, A.K.; Obradovic, Z. Genome Inform. Workshop Genome Inform. 1999, 10, 30.

(5) Ward, J.J.; Sodhi, J.S.; McGuffin, L.J.; Buxton, B.F.; Jones, D.T. J. Mol. Biol. 2004, 337, 635.

(6) Linding, R.; Jensen, L.J.; Diella, F.; Bork, P.; Gibson, T.J.; Russell, R.B. Structure 2003, 11, 1453.

(7) Pavlović-Lažetić, G.M.; Mitić, N.S.; Kovačević, J.J.; Obradović, Z.; Malkov, S.N.; Beljanski, M.V. BMC Bioinformatics 2011, 12, 66.

(8) Lupas, A.; Van Dyke, M.; Stock, J. Science 1991, 252, 1162.

(9) Broadhurst, R.W.; Nietlispach, D.; Wheatcroft, M.P.; Leadlay, P.F.; Weissman, K.J. Chem. Biol. 2003, 10, 723.

(10) Buchholz, T.J.; Geders, T.W.; Bartley F.E. $3^{\text {rd }}$; Reynolds, K.A.; Smith, J.L.; Sherman, D.H. ACS Chem. Biol. 2009, 4, 41.

(11) Sievers, F.; Wilm, A.; Dineen, D.; Gibson, T.J.; Karplus, K.; Li, W.; Lopez, R.; McWilliam, H.; Remmert, M.; Söding, J. Thompson, J.D.; Higgins, D.G. Mol. Syst. Biol. 2011, 7, 539.

(12) Whicher, J.R.; Smaga, S.S.; Hansen, D.A.; Brown, W.C.; Gerwick, W.H.; Sherman, D.H.; Smith, J.L. Chem. Biol. 2013, 20, 1340. 